\title{
Pacific
}

Journal of

Mathematics

\section{REPRESENTATIONS OF CONVEX NONDENTABLE SETS}

Spiros Argyros And Irene Deliyanni

Volume 155 No. 1 


\title{
REPRESENTATIONS OF CONVEX NON-DENTABLE SETS
}

\author{
Spiros A. Argyros AND Irene DeliyanNI
}

\begin{abstract}
It is proved that every closed convex non-R.N.P. set in a Banach space contains a non-dentable subset with a martingale coordinatization. Thus we answer affirmatively a question posed by H. Rosenthal and A. Wessel. The proof depends on the concept of the Convex Finite-Dimensional Schauder Decomposition (C.F.D.S.D.) introduced and investigated in the present paper. Certain partial positive results are also given related to the following fundamental problem: Every closed convex set either is R.N.P. or it contains a closed subset with a $\mathscr{P}$ al -representation.
\end{abstract}

Introduction. Sets with the Radon-Nikodým Property (R.N.P.) can be considered as that class of closed convex sets which enlarges the class of weakly compact sets and which still keeps most of the nice properties of the latter class. There exists an extensive literature on the study of sets with the R.N.P.; the reader should consult [Bo] and [DU]. Recently the Analytic R.N.P., the complex analogue of the R.N.P., has been studied by several authors (see [G-L-M], [G-M-S]). One of the most important problems in the theory of sets with the R.N.P. is that of the equivalence of the R.N.P. and the Krein-Milman Property (K.M.P.). In the past years several partial results have been obtained. Among them the most remarkable is W. Schachermayer's discovery that R.N.P. is equivalent to K.M.P. on strongly regular sets. Furthermore H. Rosenthal proved that every strongly regular non-R.N.P. set contains a closed convex subset on which the norm and weak topologies coincide. After all it became clear that a further study of the problem needs a further understanding of the structure of non-dentable sets, which is, of course, of independent interest.

The purpose of the present paper is to give certain representations for the elements of non-dentable closed convex subsets of Banach spaces. For this purpose we localize the concept of the FiniteDimensional Schauder Decomposition (F.D.S.D.) by defining the Convex Finite-Dimensional Schauder Decomposition (C.F.D.S.D.). This allows us to express uniquely the elements of certain convex sets as norm-converging series in a similar way as is done in spaces with a 
F.D.S.D. We also show that for convex sets failing the R.N.P. and belonging to certain classes we can find subsets of them with a "complete structure" which is described as a $\mathscr{P}$ al -representation.

The paper is organized into five sections each one containing the following:

In $\S 0$ we recall the definitions and some properties of $\delta$-approximate bushes and regular $\delta$-bushes.

Section 1 is devoted to the definition of $\delta$-approximate bushes $\left(x_{\alpha}\right)_{\alpha \in \mathscr{A}}$ defining a C.F.D.S.D. and to the study of the properties that this notion induces on the set $K=\overline{\mathrm{co}}\left(\tilde{x}_{\alpha}\right)_{\alpha \in \mathscr{A}}$, where $\left(\tilde{x}_{\alpha}\right)_{\alpha \in \mathscr{A}}$ is the averaged back bush corresponding to $\left(x_{\alpha}\right)_{\alpha \in \mathscr{A}}$. The most important is that each element of $K$ has a unique expansion as

$$
x=\sum_{n=0}^{\infty} \sum_{|\alpha|=n} \lambda_{\alpha}^{(x)} y_{\alpha}
$$

where $\left(y_{\alpha}\right)_{\alpha \in \mathscr{A}}$ are the nodes of $\left(x_{\alpha}\right)_{\alpha \in \mathscr{A}}$ and

$$
\lambda_{\alpha}^{(x)} \geq 0, \quad \lambda_{0}^{(x)}=1, \quad \lambda_{\alpha}^{(x)}=\sum_{\beta \in S_{\alpha}} \lambda_{\beta}^{(x)} .
$$

As we show, this expansion is equivalent to the notion of a martingale coordinatization introduced by $\mathbf{H}$. Rosenthal and A. Wessel in [R-W].

The next two sections are mainly devoted to the proof of the following result:

THeOREM A. If $L$ is a closed convex non-R.N.P. subset of a Banach space $X$ then it contains a $\delta$-approximate bush $\left(x_{\alpha}\right)_{\alpha \in \mathscr{A}}$ defining a C.F.D.S.D. Hence it contains a closed convex subset $K=\overline{\mathrm{co}}\left(\tilde{x}_{\alpha}\right)_{\alpha \in \mathscr{A}}$ such that each $x$ in $K$ is expressed uniquely as

$$
x=\sum_{n=0}^{\infty} \sum_{|\alpha|=n} \lambda_{\alpha}^{(x)} y_{\alpha}
$$

The proof of this theorem is divided in two parts. The first, given in $\S 2$ (Theorem 2.3), contains the case of closed convex sets $L$ failing the Point of Continuity Property (P.C.P.) and the second, given in $\S 3$ (Theorem 3.7), concerns non-R.N.P. sets with the P.C.P. In Theorem 3.7 we also prove that the chosen set $K$ satisfies the additional property that the norm and weak topologies on it coincide.

Further in $\S 2$ we prove two more results concerning sets which fail the P.C.P., Theorem 2.7 and Theorem 2.13, which we use in $\S 4$ to establish $\mathscr{P} a l$-representations in certain convex sets. 
Section 4 contains results concerning $\mathscr{P}$ al -representations. We define $\mathscr{P} a l$ to be the closed convex subset of $M[0,1]$ consisting of all probability atomless measures. A closed convex set $K$ in a Banach space $X$ has a $\mathscr{P} a l$-representation if there exists an affine, continuous, onto injection $T: \mathscr{P a l} \rightarrow K$. It is an easy observation that if $K$ has a $\mathscr{P} a l$-representation then it has no extreme points, hence it fails the K.M.P. The problem whether every non-R.N.P. set contains a closed convex subset with a $\mathscr{P} a l$-representation was posed to the first author by Professor Rosenthal. The main result of $\S 4$ gives a partial positive answer to this problem. More precisely we show:

THeOREM B. Let $L$ be a closed convex non-R.N.P. subset of a Banach space $X$ such that one of the following conditions is satisfied:

(i) The R.N.P. is not equivalent to the P.C.P. on the subsets of $L$,

(ii) $X$ has an unconditional basis,

(iii) $L$ is a subset of the positive cone of $L^{1}[0,1]$.

Then there exists a closed convex subset $K$ of $L$ with a $\mathscr{P} a l$-representation.

As we mentioned before Theorem B implies that the R.N.P. is equivalent to the K.M.P. on $L$, if $L$ satisfies (i), (ii) or (iii). This is already known from earlier works of Schachermayer [S2], Rosenthal $[\mathbf{R}]$, Rosenthal and Wessel $[\mathbf{R}-\mathbf{W}]$ and Caselles $[\mathbf{C}]$. As a corollary of Theorem B we get that every non-R.N.P. $w^{*}$-closed convex subset of a dual Banach space $X$ contains a separable subset with a $\mathscr{P}_{a}$ representation.

0. Approximate bushes. In this section we recall the definition of a $\delta$-approximate bush and the corresponding averaged back (regular) bush.

0.1 . Notation. We denote by $N^{(N)}$ the set of all finite sequences of natural numbers of the form $\left(0, a_{1}, a_{2}, \ldots, a_{n}\right)$. For $\alpha \in N^{(N)}$ we denote by $|\alpha|$ the length of $\alpha$ and for convenience we set $|(0)|=0$ and $\left|\left(0, a_{1}, \ldots, a_{n}\right)\right|=n$. For $\alpha \in N^{(N)}, n \in N, n \leq|\alpha|$ we denote by $\alpha \mid n$ the restriction of $\alpha$ on the set $\{0, \ldots, n\}$ and for $\alpha, \beta \in N^{(N)}$ we define by $\alpha \leq \beta$ iff $|\alpha| \leq|\beta|$ and $\beta|| \alpha \mid=\alpha$ which clearly defines a partial order on $N^{(N)}$.

$A$ finitely branching tree, denoted by $\mathscr{A}$, is a subset of $N^{(N)}$ such that for every $\alpha \in \mathscr{A}$ and $n \in N$ with $n \leq|\alpha|, \alpha \mid n \in \mathscr{A}$ and for every $n \in N$ the set $\mathscr{A}_{n}=\{\alpha \in \mathscr{A}:|\alpha|=n\}$ is finite. If $\mathscr{A}$ is a 
finitely branching tree and $\alpha \in \mathscr{A}$ we denote by

$$
S_{\alpha}=\{\beta: \alpha<\beta,|\beta|=|\alpha|+1\} .
$$

0.2. Definition. (i) A bounded subset $\left(x_{\alpha}\right)_{\alpha \in \mathscr{A}}$ of a Banach space $X$ is said to be a $\delta$-approximate bush with $\delta>0$ iff $\mathscr{A}$ is a finitely branching tree, for all $\alpha \in \mathscr{A}, \beta \in S_{\alpha},\left\|x_{\alpha}-x_{\beta}\right\|>\delta$ and there exists $\left\{\lambda_{\beta}: \beta \in S_{\alpha}\right\}$ with $\lambda_{\beta} \geq 0, \sum_{\beta \in S_{\alpha}} \lambda_{\beta}=1$ and $\left\|x_{\alpha}-\sum_{\beta \in S_{\alpha}} \lambda_{\beta} x_{\beta}\right\|<$ $\varepsilon_{|\alpha|}$ where $\sum_{n=0}^{\infty} \varepsilon_{n}<\frac{\delta}{4}$.

(ii) A set $\left(x_{\alpha}\right)_{\alpha \in \mathscr{A}}$, as before, is said to be a $\delta$-bush iff for $\alpha \in \mathscr{A}$, $\beta \in S_{\alpha},\left\|x_{\alpha}-x_{\beta}\right\|>\delta$ and for all $\alpha \in \mathscr{A}, x_{\alpha}=\sum_{\beta \in S_{\alpha}} \lambda_{\beta} x_{\beta}$ for some $\left\{\lambda_{\beta}: \beta \in S_{\alpha}\right\}, \lambda_{\beta} \geq 0, \sum_{\beta \in S_{\alpha}}, \lambda_{\beta}=1$.

The averaged back bush corresponding to an approximate $\delta$-bush.

Let $\left(x_{\alpha}\right)_{\alpha \in \mathscr{A}}$, be an approximate $\delta$-bush. For all $\alpha \in \mathscr{A}, m>|\alpha|$ we define $x_{\alpha}^{m}=\sum_{|\beta|=m} \lambda_{\beta}^{(\alpha)} x_{\beta}$ to be a convex combination defined inductively as follows: For $m=|\alpha|+1, x_{\alpha}^{m}=\sum_{\beta \in S_{\alpha}} \lambda_{\beta} x_{\beta}$ where $\left\{\lambda_{\beta}: \beta \in S_{\alpha}\right\}$ is the set which appeared in the definition of the $\delta$ approximate bush. Suppose that for some $n$ the set $\left\{\lambda_{\beta}^{(\alpha)}:|\beta|=n\right\}$ has been defined; we define $\lambda_{\gamma}^{(\alpha)}=\lambda_{\beta}^{(\alpha)} \lambda_{\gamma}$ where $|\gamma|=n+1, \gamma \in S_{\beta}$ and $\lambda_{\gamma}$ is the coefficient appearing in the definition of the approximate bush.

The following properties are easily established:

(i) For all $\alpha \in \mathscr{A}$ the sequence $\left\{x_{\alpha}^{m}\right\}_{m>|\alpha|}$ is norm Cauchy.

(ii) If $\tilde{x}_{\alpha}=\lim _{m \rightarrow \infty} x_{\alpha}^{m}$ then for $\alpha \in \mathscr{A}, \beta \in S_{\alpha},\left\|\tilde{x}_{\alpha}-\tilde{x}_{\beta}\right\|>\frac{\delta}{2}$ and

$$
\tilde{x}_{\alpha}=\sum_{\beta \in S_{\alpha}} \lambda_{\beta} \tilde{x}_{\beta} .
$$

(iii) Every $\tilde{x}_{\alpha}$ belongs to $\overline{\mathrm{co}}\left(x_{\alpha}\right)_{\alpha \in \mathscr{A}}$.

0.3. Notation. If $\left(x_{\alpha}\right)_{\alpha \in \mathscr{A}}$ is a $\delta$-approximate bush we set $y_{0}=$ $x_{0}$ and for $\alpha \in \mathscr{A}, y_{\beta}=x_{\beta}-x_{\alpha}$ for all $\beta \in S_{\alpha}$. The vectors $\left(y_{\alpha}\right)_{\alpha \in \mathscr{A}}$ are called the nodes of the approximate bush.

0.4. Remarks. An easy computation shows the following identity. For every convex combination $\sum_{|\beta|=m} \lambda_{\beta} x_{\beta}$ we have

$$
\sum_{|\beta|=m} \lambda_{\beta} x_{\beta}=\sum_{n=0}^{m} \sum_{|\alpha|=n} \mu_{\alpha} y_{\alpha}
$$

where $\mu_{\alpha}=\lambda_{\alpha}$ for $|\alpha|=m$ and if $|\alpha|<m, \mu_{\alpha}=\sum_{\gamma \in S_{\alpha}} \mu_{\gamma}$. 
0.5. Definition. Let $\left(x_{\alpha}\right)_{\alpha \in \mathscr{A}}$ be a bush of elements of a Banach space $X$. The bush function of $\left(x_{\alpha}\right)_{\alpha \in \mathscr{A}}$ is a family $\left(\lambda_{\alpha}\right)_{\alpha \in \mathscr{A}}$ such that $\lambda_{\alpha} \geq 0, \sum_{\beta \in S_{\alpha}} \lambda_{\beta}=1$ and $x_{\alpha}=\sum_{\beta \in S_{\alpha}} \lambda_{\beta} x_{\beta}$.

If $\left(x_{\alpha}\right)_{\alpha \in \mathscr{A}}$ is a $\delta$-approximate bush we define the bush-function of it to be that of the averaged back bush $\left(\tilde{x}_{\alpha}\right)_{\alpha \in \mathscr{A}}$.

REMARK 0.6. Let $\left(y_{\alpha}\right)_{\alpha \in \mathscr{A}}$ be the nodes of the $\delta$-approximate bush $\left(x_{\alpha}\right)_{\alpha \in \mathscr{A}}$ and $\left(\tilde{y}_{\alpha}\right)_{\alpha \in \mathscr{A}}$ the nodes of the corresponding regular bush $\left(\tilde{x}_{\alpha}\right)_{\alpha \in \mathscr{A}}$, then for any family $\left(\mu_{\alpha}\right)_{\alpha \in \mathscr{A}}$ of real numbers such that $\mu_{0}=$ $1, \mu_{\alpha} \geq 0, \mu_{\alpha}=\sum_{\beta \in S_{\alpha}} \mu_{\beta}$ (a normalized conditionally determined family, following $[\mathbf{R}-\mathbf{W}])$ we have

$$
\sum_{n=0}^{\infty} \sum_{|\beta|=n} \mu_{\beta} y_{\beta}=\sum_{n=0}^{\infty} \sum_{|\beta|=n} \mu_{\beta} \tilde{y}_{\beta}
$$

whenever either series converges.

This follows by Remark 0.4 and the fact

$$
\left\|\sum_{|\alpha|=k} \mu_{\alpha} x_{\alpha}-\sum_{|\alpha|=k} \mu_{\alpha} \tilde{x}_{\alpha}\right\| \leq \sum_{\rho \geq k} \varepsilon_{\rho} .
$$

1. The convex finite dimensional Schauder decomposition (C.F.D.S.D.).

1.1. Definition. A $\delta$-approximate bush $\left(x_{\alpha}\right)_{\alpha \in \mathscr{A}}$ defines a C.F.D.S.D. if the following conditions are satisfied:

(i) The nodes $\left(y_{\alpha}\right)_{\alpha \in \mathscr{A}}$ are linearly independent.

(ii) There exists $k>0$ such that for $n<m, \sum_{|\alpha|=m} \lambda_{\alpha} x_{\alpha}$ an absolutely convex combination we have

$$
\left\|\sum_{|\beta|=n} \mu_{\beta} x_{\beta}\right\| \leq k \cdot\left\|\sum_{|\alpha|=m} \lambda_{\alpha} x_{\alpha}\right\|
$$

where

$$
\mu_{\beta}=\sum_{|\alpha|=m, \beta<\alpha} \lambda_{\alpha} .
$$

1.2. REMARK. We note that for $n<m$ the map

$$
P_{m, n}: \sum_{|\alpha|=m} \lambda_{\alpha} x_{\alpha} \rightarrow \sum_{|\beta|=n} \mu_{\beta} x_{\beta}
$$

where $\mu_{\beta}$ is as before, is well-defined and linear. Indeed by using the 
alternative expression of $\sum_{|\alpha|=m} \lambda_{\alpha} x_{\alpha}$ we get that

$$
P_{m, n}\left[\sum_{k=0}^{m} \sum_{|\gamma|=k} \xi_{\gamma} y_{\gamma}\right]=\sum_{k=0}^{n} \sum_{|\gamma|=k} \xi_{\gamma} y_{\gamma}
$$

which clearly is well-defined and linear because of the linear independence of the vectors $\left(y_{\alpha}\right)_{\alpha \in \mathscr{A}}$. It is also obvious that for $m_{1}>m_{2}>n$

$$
P_{m_{1}, n}=P_{m_{2}, n} \circ P_{m_{1}, m_{2}} .
$$

Hence we will use the notation $P_{n}$ for any $P_{m, n}$.

1.3. Proposition. Let $\left(x_{\alpha}\right)_{\alpha \in \mathscr{A}}$ be a $\delta$-approximate bush defining a C.F.D.S.D. and $\left(\tilde{x}_{\alpha}\right)_{\alpha \in \mathscr{A}}$ the averaged back bush. Then for every $x \in \overline{\mathrm{co}}\left(\tilde{x}_{\alpha}\right)_{\alpha \in \mathscr{A}}$ we have that $x$ has a unique expression:

$$
x=\sum_{n=0}^{\infty} \sum_{|\alpha|=n} \lambda_{\alpha}^{(x)} y_{\alpha}
$$

where $\left(y_{\alpha}\right)_{\alpha \in \mathscr{A}}$ are the nodes of $\left(x_{\alpha}\right)_{\alpha \in \mathscr{A}}$ and $\lambda_{0}^{(x)}=1,0 \leq \lambda_{\alpha}^{(x)} \leq 1$, $\lambda_{\alpha}^{(x)}=\sum_{\beta \in S_{\alpha}} \lambda_{\beta}^{(x)}$.

Proof. We note first that if $x \in \overline{\operatorname{co}}\left(\tilde{x}_{\alpha}\right)_{\alpha \in \mathscr{A}}$ and $x$ has an expression of the above form then it is unique.

Indeed, if

$$
\sum_{n=0}^{\infty} \sum_{|\alpha|=n} \mu_{\alpha}^{(x)} y_{\alpha}=\sum_{n=0}^{\infty} \sum_{|\alpha|=n} \lambda_{\alpha}^{(x)} y_{\alpha}
$$

and there exists $\alpha_{0} \in \mathscr{A}$ with $\lambda_{\alpha_{0}}^{(x)} \neq \mu_{\alpha_{0}}^{(x)}$, suppose $\left|\alpha_{0}\right|=n_{0}$ and choose $\varepsilon>0$ such that

$$
\left\|\sum_{n=0}^{n_{0}} \sum_{|\alpha|=n} \lambda_{\alpha}^{(x)} y_{\alpha}-\sum_{n=0}^{n_{0}} \sum_{|\alpha|=n} \mu_{\alpha}^{(x)} y_{\alpha}\right\|>\varepsilon .
$$

Choose $m \in N$ such that

$$
\left\|\sum_{n=0}^{m} \sum_{|\alpha|=n} \lambda_{\alpha}^{(x)} y_{\alpha}-\sum_{n=0}^{m} \sum_{|\alpha|=n} \mu_{\alpha}^{(x)} y_{\alpha}\right\|<\frac{\varepsilon}{k}
$$

where $k>0$ is the constant for the C.F.D.S.D. Then applying $P_{n_{0}}$ we get

$$
\left\|P_{n_{0}}\left(\sum_{n=0}^{m} \sum_{|\alpha|=n} \lambda_{\alpha}^{(x)} y_{\alpha}-\sum_{n=0}^{m} \sum_{|\alpha|=n} \mu_{\alpha}^{(x)} y_{\alpha}\right)\right\|<k \cdot \frac{\varepsilon}{k}=\varepsilon
$$

which contradicts $(*)$, proving our claim. 
Thereafter it suffices to show that every $x \in \overline{c o}\left(\tilde{x}_{\alpha}\right)_{\alpha \in \mathscr{A}}$ has at least one such representation.

Indeed $\tilde{x}_{\alpha}$ is by definition equal to $\sum_{n=0}^{\infty} \sum_{|\beta|=k} \mu_{\beta} \tilde{y}_{\beta}$, where for $\beta>\alpha, \mu_{\beta}=\prod_{\alpha<\gamma \leq \beta} \lambda_{\gamma}$, with $\left(\lambda_{\alpha}\right)_{\alpha \in \mathscr{A}}$ the bush function of $\left(x_{\alpha}\right)_{\alpha \in \mathscr{A}}$, and $\mu_{\beta}=1$, for $\beta \leq \alpha$, and $\mu_{\beta}=0$, for $\beta$ incomparable to $\alpha$. Now by Remark 0.6 we get that $\tilde{x}_{\alpha}$ has a representation of the desired form, and this is extended to the elements of $\operatorname{co}\left(x_{\alpha}\right)_{\alpha \in \mathscr{A}}$.

We define

$$
P_{n}: \operatorname{co}\left(\tilde{x}_{\alpha}\right)_{\alpha \in \mathscr{A}} \rightarrow \operatorname{co}\left(x_{\alpha}\right)_{|\alpha|=n}
$$

by the rule

$$
P_{n}\left(\sum_{n=0}^{\infty} \sum_{|\alpha|=k} \lambda_{\alpha}^{(x)} y_{\alpha}\right)=\sum_{n=0}^{n} \sum_{|\alpha|=k} \lambda_{\alpha}^{(x)} y_{\alpha}
$$

and we claim that $\left\|P_{n}(x)-P_{n}(y)\right\| \leq k\|x-y\|$.

We observe that $\lim _{m \rightarrow \infty} P_{m}(x)=x$ and if $n<m, P_{n}\left(P_{m}(x)\right)=$ $P_{n}(x)$. Hence

$$
\lim _{m \rightarrow \infty}\left\|P_{m}(x)-P_{m}(y)\right\|=\|x-y\|
$$

and

$$
\begin{aligned}
\left\|P_{n}(x)-P_{n}(y)\right\| & =\left\|P_{n}\left(P_{m}(x)\right)-P_{n}\left(P_{m}(y)\right)\right\| \\
& \leq k\left\|P_{m}(x)-P_{m}(y)\right\|
\end{aligned}
$$

and finally we get

$$
\left\|P_{n}(x)-P_{n}(y)\right\| \leq k\|x-y\| .
$$

Therefore we can extend $P_{n}$ on the set $\overline{\mathrm{co}}\left(\tilde{x}_{\alpha}\right)_{\alpha \in \mathscr{A}}$. Clearly for $x \in \overline{\mathrm{co}}\left(\tilde{x}_{\alpha}\right)_{\alpha \in \mathscr{A}}$ we have $P_{n}\left(P_{m}(x)\right)=P_{n}(x)$ for all $m \in N, n<m$ and easily we get a representation for any $x$.

1.4. REMARK. (i) Our goal is to show that every closed convex bounded non-dentable subset of a Banach space $X$ contains a $\delta$ approximate bush defining a C.F.D.S.D. Bourgain has shown that every non-R.N.P. Banach space contains a subspace with a F.D.S.D. failing the R.N.P. [B]. Our result can be considered as a localization of Bourgain's Theorem. We will distinguish two cases. The first is of sets without the P.C.P. and the second is of non-dentable sets with the P.C.P.

(ii) Proposition 1.3 and Remark 0.6 imply that if $\left(x_{\alpha}\right)_{\alpha \in \mathscr{A}}$ is a $\delta$ approximate bush which defines a C.F.D.S.D. then the averaged back bush $\left(\tilde{x}_{\alpha}\right)_{\alpha \in \mathscr{A}}$ is a martingale coordinatization for its closed convex 
hull. We recall that a bush $\left(x_{\alpha}\right)_{\alpha \in \mathscr{A}}$ is a martingale coordinatization ([R-W]) if each $x$ in the closed convex hull of $\left(x_{\alpha}\right)_{\alpha \in \mathscr{A}}$ has a unique representation of the form $\sum_{n=0}^{\infty} \sum_{|\alpha|=n} \lambda_{\alpha}^{(x)} y_{\alpha}$ where $\left(y_{\alpha}\right)_{\alpha \in \mathscr{A}}$ are the nodes of the bush. A restatement of our result is that every nonR.N.P. set in a Banach space $X$ contains a $\delta$-bush $\left(x_{\alpha}\right)_{\alpha \in \mathscr{A}}$ which is a martingale coordinatization. This answers affirmatively a question posed in the final Remark of [R-W].

Next we give a criterion for the existence of $\delta$-approximate bushes defining a C.F.D.S.D.

1.5. LemMA. Let $\left(x_{\alpha}\right)_{\alpha \in \mathscr{A}}$ be a $\delta$-approximate bush such that:

(i) $\left(x_{\alpha}\right)_{\alpha \in \mathscr{A}}$ are linearly independent.

(ii) For all $n \in N$ there exists a subset $F_{n}$ of $B_{X^{*}}\left(1-\varepsilon_{n}\right)$-norming the absolutely convex hull of $\left\{x_{\alpha}:|\alpha|=n\right\}$ and there exists $\bar{\delta}_{n}$ such that

$$
\prod_{n=0}^{\infty}\left(1-\bar{\delta}_{n}-\varepsilon_{n}\right)^{-1}<\infty
$$

and setting

$$
\delta_{n}=\inf \left\{\left\|\sum_{|\alpha|=n} \lambda_{\alpha} x_{\alpha}\right\|: \sum_{|\alpha|=n}\left|\lambda_{\alpha}\right|=1\right\} \cdot \bar{\delta}_{n}
$$

we have

$$
\left\|f\left(x_{\alpha}-x_{\beta}\right)\right\|<\delta_{n}
$$

for all $f \in F_{n}, \alpha \in \mathscr{A}_{n}, \beta \in S_{\alpha}$.

(iii) For all $\alpha \in \mathscr{A}_{n}, f \in F_{n}$ the set $\left\{f\left(x_{\alpha}-x_{\beta}\right): \beta \in S_{\alpha}\right\}$ is a singleton.

Then $\left(x_{\alpha}\right)_{\alpha \in \mathscr{A}}$ defines a C.F.D.S.D.

Proof. We claim that for $n \in N$ and any linear combination $\sum_{|\alpha|=n+1} \lambda_{\alpha} x_{\alpha}$ we have

$$
\left\|P_{n}\left(\sum_{|\alpha|=n+1} \lambda_{\alpha} x_{\alpha}\right)\right\| \leq\left(1-\varepsilon_{n}-\bar{\delta}_{n}\right)^{-1}\left\|\sum_{|\alpha|=n+1} \lambda_{\alpha} x_{\alpha}\right\| .
$$

Indeed, notice that

$$
P_{n}\left(\sum_{|\alpha|=n+1} \lambda_{\alpha} x_{\alpha}\right)=\sum_{|\beta|=n}\left(\sum_{\alpha \in S_{\beta}} \lambda_{\alpha}\right) x_{\beta}
$$


and choose $f \in F_{n}\left(1-\varepsilon_{n}\right)$-norming the vector $P_{n}\left(\sum_{|\alpha|=n+1} \lambda_{\alpha} x_{\alpha}\right)$. Then we have

$$
\begin{aligned}
& \left\|\sum_{|\alpha|=n+1} \lambda_{\alpha} x_{\alpha}\right\| \geq\left|f\left(\sum_{|\alpha|=n+1} \lambda_{\alpha} x_{\alpha}\right)\right| \\
& \geq\left|f\left(\sum_{|\beta|=n}\left(\sum_{\alpha \in S_{\beta}} \lambda_{\alpha}\right) x_{\beta}\right)\right|-\sum_{|\beta|=n}\left|\sum_{\alpha \in S_{\beta}} \lambda_{\alpha} f\left(x_{\alpha}-x_{\beta}\right)\right| \\
& \geq\left(1-\varepsilon_{n}\right)\left\|P_{n}\left(\sum_{|\alpha|=n+1} \lambda_{\alpha} x_{\alpha}\right)\right\|-\sum_{|\beta|=n}\left|\sum_{\alpha \in S_{\beta}} \lambda_{\alpha}\right| \cdot \delta_{n} \\
& \geq\left(1-\varepsilon_{n}\right)\left\|P_{n}\left(\sum_{|\alpha|=n+1} \lambda_{\alpha} x_{\alpha}\right)\right\|-\left\|P_{n}\left(\sum_{|\alpha|=n+1} \lambda_{\alpha} x_{\alpha}\right)\right\| \cdot \bar{\delta}_{n} \\
& =\left(1-\varepsilon_{n}-\bar{\delta}_{n}\right) \cdot\left\|P_{n}\left(\sum_{|\alpha|=n+1} \lambda_{\alpha} x_{\alpha}\right)\right\| .
\end{aligned}
$$

So the claim is proved. We set $K=\prod_{n=0}^{\infty}\left(1-\varepsilon_{n}-\bar{\delta}_{n}\right)^{-1}$ and we easily verify that for $n<m$

and the proof is complete.

$$
\left\|P_{n}\left(\sum_{|\alpha|=m} \lambda_{\alpha} x_{\alpha}\right)\right\| \leq K \cdot\left\|\sum_{|\alpha|=m} \lambda_{\alpha} x_{\alpha}\right\|
$$

The next lemma is crucial for the construction of approximate bushes defining a C.F.D.S.D. In particular it will ensure the condition (iii) of the previous lemma.

1.6. LEMMA. Let $K$ be a convex closed bounded subset of a Banach space $X, f_{1}, \ldots, f_{n}$ elements of $X^{*}$ and $\varepsilon$ a real number with $0<$ $\varepsilon<1$.

Then there exist a finite dimensional subspace $Y$ of $X$ and $a \delta>0$ such that for every $x_{0} \in K$ there exists a set of reals $\left(\mathscr{A}_{i}\left(x_{0}\right)\right)_{i=1}^{n}$ such that for every $x \in K$ with $\left|f_{i}\left(x-x_{0}\right)\right|<\delta$ for all $i=1, \ldots, n$ there exists $z \in Y \cap K$ with

$$
f_{i}((1-\varepsilon) x+\varepsilon z)=\mathscr{A}_{i}+f_{i}\left(x_{0}\right) .
$$

Proof. We can suppose that $0 \in K$ and $f_{1}, \ldots, f_{n}$ restricted on $K$ are linearly independent. We set

$$
\vec{f}: K \rightarrow R^{n}
$$


defined by

$$
\vec{f}(x)=\left(f_{1}(x), \ldots, f_{n}(x)\right) .
$$

It is clear that $\vec{f}[K]$ has interior in $R^{n}$.

Select a finite dimensional subspace $Y$ of $X$ such that $\vec{f}[Y \cap K]$ contains a set of the form $\prod_{i=1}^{n}\left[a_{i}, b_{i}\right]$ with $a_{i}<b_{i}$.

Set $\delta=\varepsilon \cdot \min \left\{\frac{b_{i}-a_{1}}{2} 1 \leq i \leq n\right\}$ and for $x_{0} \in K$ define

$$
A_{i}=\varepsilon\left(\frac{a_{i}+b_{i}}{2}-f_{i}\left(x_{0}\right)\right) \text {. }
$$

Suppose that $x \in K$ and it satisfies

$$
\left|f_{i}\left(x-x_{0}\right)\right|<\delta \text { for } i=1, \ldots, n .
$$

The desired $z$ will exist provided that

$$
\begin{aligned}
a_{i}< & f_{i}(z)=\frac{A_{i}+f_{i}\left(x_{0}\right)-(1-\varepsilon) f_{i}(x)}{\varepsilon}<b_{i} \\
\Leftrightarrow & \varepsilon a_{i}<A_{i}+f_{i}\left(x_{0}\right)-(1-\varepsilon) f_{i}(x)<\varepsilon b_{i} \\
\Leftrightarrow & \varepsilon \alpha_{i}<A_{i}+\varepsilon f_{i}\left(x_{0}\right)+(1-\varepsilon)\left(f_{i}\left(x_{0}\right)-f_{i}(x)\right)<\varepsilon b_{i} \\
\Leftrightarrow & \varepsilon a_{i}<\varepsilon \frac{a_{i}+b_{i}}{2}-\varepsilon f_{i}\left(x_{0}\right)+\varepsilon f_{i}\left(x_{0}\right) \\
& +(1-\varepsilon)\left(f_{i}\left(x_{0}\right)-f_{i}(x)\right)<\varepsilon b_{i}
\end{aligned}
$$

but the last relation follows from the fact

$$
(1-\varepsilon)\left|f_{i}\left(x_{0}-x\right)\right|<\delta \leq \varepsilon \cdot \frac{b_{i}-a_{i}}{2}
$$

The proof is complete.

1.7. Definition. Let $\left(x_{\alpha}\right)_{\alpha \in \mathscr{A}}$ be a $\delta$-approximate bush defining a C.F.D.S.D. Then for each $\alpha \in \mathscr{A}$ we define the coordinate functional $f_{\alpha}: \overline{\operatorname{co}}\left(\tilde{x}_{\alpha}\right)_{\alpha \in \mathscr{A}} \rightarrow R$ by the rule

$$
f_{\alpha}\left(\sum_{n=0}^{\infty} \sum_{|\beta|=n} \lambda_{\beta}^{(x)} y_{\beta}\right)=\lambda_{\alpha}^{(x)} .
$$

1.8. Remark. Since the projections $\left(P_{n}\right)_{n \in N}$ are norm continuous we get that each $f_{\alpha}$ is also norm continuous.

1.9. LeMmA. The coordinate functionals $\left(f_{\alpha}\right)_{\alpha \in \mathscr{A}}$ are weakly continuous on $\overline{\mathrm{co}}\left(\tilde{x}_{\alpha}\right)_{\alpha \in \mathscr{A}}$.

Proof. Suppose that some $f_{\alpha}$ is not weakly continuous. Then there exists $x$ and a net $\left(x_{i}\right)_{i \in I}$ weakly convergent to $x$ such that $\left(f_{\alpha}\left(x_{i}\right)\right)_{i \in I}$ 
does not converge to $f(x)$. Hence there exists a subnet $\left(x_{i}\right)_{j \in J}$ and $\varepsilon>0$ such that either

$$
f_{\alpha}(x)+\varepsilon<f_{\alpha}\left(x_{i_{j}}\right) \quad \text { or } \quad f_{\alpha}(x)-\varepsilon>f_{\alpha}\left(x_{i_{j}}\right) .
$$

Assume that the first holds. Then for any convex combination

$$
\sum_{s=1}^{l} \alpha_{s} x_{i_{s}}=y
$$

we have that

$$
f_{\alpha}(x)+\varepsilon<f_{\alpha}(y) .
$$

But from Mazur's Theorem we have that there exists a sequence of convex combinations norm convergent to $x$. This is a contradiction proving the result.

2. The C.F.D.S.D. for convex sets without the P.C.P. This section is devoted to the proof of the existence of approximate bushes defining a C.F.D.S.D. into any convex set without the Point of Continuity Property.

We begin by recalling the definition of the P.C.P.

2.1. Definition. A subset $K$ of a Banach space $X$ has the Point of Continuity Property (P.C.P.) iff for every $L$ subset of $K$ and $\varepsilon>0$ there exists a relatively weakly open subset $W$ of $L$ with $W \neq \varnothing$ and $\operatorname{diam}[W]<\varepsilon$.

2.2. Lemma. Let $K$ be a convex bounded closed separable set without the P.C.P. Then there exist a subset $D$ of $K$ consisting of linearly independent vectors and $\delta>0$ such that every weakly relatively open subset $W$ of $D$ has $\operatorname{diam}[W]>\delta$.

Proof. We first notice that Baire's Theorem ensures the existence of an uncountable subset $D_{1}$ of $K$ consisting of linearly independent vectors. Also there exists a subset $E$ of $K$ and $\delta_{1}>0$ such that every weakly relatively open subset $W$ of $E$ has $\operatorname{diam}[W]>\delta_{1}$. Further, due to the countable tightness of the weak topology, we may assume that $E$ is of the form $E=\left\{x_{s}: s \in N^{(N)}\right\}$ where $x_{s}$ is a weak limit of the set $\left\{x_{t}: t \in S_{s}\right\}$ and $\left\|x_{s}-x_{t}\right\|>\delta_{1}$ for all $s \in N^{(N)}, t \in S_{s}$. Next using transfinite induction, we choose an uncountable subset $D_{2}$ of $D_{1}$ such that the elements of $D_{2}$ are linearly independent over the linear span of $E$. 
Since $K$ is separable there exists a subset $D_{2}^{\prime}$ of $D_{2}$ such that $D_{2}^{\prime}=\left\{y_{s}: s \in N^{(N)}\right\}$ where $y_{s} \neq y_{t}$ for $s \neq t$ and $y_{s}$ is the norm limit of $y_{(s, n)}$.

Consider the set

$$
L=\left\{z_{s}=\frac{x_{s}+y_{s}}{2}: s \in N^{(N)}\right\} .
$$

It is easy to check that $L$ consists of linearly independent vectors and every relatively weakly open subset $W$ of $L$ contains some $z_{s}$, $z_{(s, n)}$ such that

$$
\left\|z_{s}-z_{(s, n)}\right\|>\frac{\delta_{1}}{4}=\delta .
$$

This completes the proof of the lemma.

We are ready now to give the statement and the proof of the main result of this section.

2.3. TheOREM. Let $K$ be a separable closed convex set contained in $B_{X}$ and failing the P.C.P. Then for some $\delta^{\prime}$ the set $K$ contains $a$ $\delta^{\prime}$-approximate bush $\left(w_{\alpha}\right)_{\alpha \in \mathscr{A}}$ which defines a C.F.D.S.D.

Proof. Fix a set $D \subset K$ and a $\delta>0$ satisfying the conclusion of Lemma 1.

Fix also two sequences of positive reals $\left\{\varepsilon_{n}\right\}_{n \in N},\left\{\rho_{n}\right\}_{n \in N}$ with $\varepsilon_{n}<\frac{1}{2}, 1-\frac{\delta}{2^{n} \cdot 100}<\rho_{n}<1$ for all $n$, and

$$
\prod_{n=0}^{\infty}\left(1-2 \varepsilon_{n}\right)>0, \quad \prod_{n=1}^{\infty} \rho_{n} \geq \frac{1}{2} .
$$

Inductively we shall construct the following: A finitely branching tree $\mathscr{A}$. Two families of vectors $\left(x_{\alpha}\right)_{\alpha \in \mathscr{A}}$ and $\left(z_{\alpha}\right)_{\alpha \in \mathscr{A}}$ both contained in $K$. A bush function $\left(\lambda_{\alpha}\right)_{\alpha \in \mathscr{A}}$. A sequence $\left\{F_{n}\right\}_{n \in N}$ of finite subsets of $B_{X^{*}}$. A sequence $\left\{\mu_{n}\right\}_{n=1}^{\infty}$ of positive reals having the following properties:

For all $n \in N$ and for all $\alpha \in \mathscr{A}$

(i) $x_{\alpha} \in D$.

(ii) If $|\alpha|=n$ then $\left\|\sum_{\beta \in S_{\alpha}} \lambda_{\beta} x_{\beta}-x_{\alpha}\right\|<\frac{\delta}{50} \cdot \frac{1}{2^{n+1}}$.

(iii) For all $\beta \in S_{\alpha},\left\|x_{\alpha}-x_{\beta}\right\|>\frac{\delta}{2}$.

(iv) The vectors $\left\{x_{\alpha}\right\}_{|\alpha|=n}$ are linearly independent over the linear span of

$$
\left\{x_{\beta}:|\beta|<n\right\} \cup\left\{z_{\beta}:|\beta| \leq n\right\} .
$$


(v) $\max _{f \in F_{n}} f(y) \geq\left(1-\varepsilon_{n}\right)\|y\|$ holds for all

$$
y \in\left\langle\left\{x_{\alpha}:|\alpha|=n\right\} \cup\left\{z_{\beta}:|\beta| \leq n\right\}\right\rangle .
$$

(vi) Set

$$
a_{n}:=\inf \left\{\left\|\sum_{|\alpha|=n} t_{\alpha} x_{\alpha}+y\right\|: \sum_{|\alpha|=n}\left|t_{\alpha}\right|=1, y \in\left\langle z_{\beta}:|\beta| \leq n\right\rangle\right\} \text {. }
$$

By (i) $a_{n}$ is strictly positive. The real $\mu_{n+1}$ is chosen so that

$$
0<1-\mu_{n+1} \leq \min \left\{\frac{a_{n} \cdot \varepsilon_{n}}{2}, 1-\rho_{n+1}\right\} \text {. }
$$

Furthermore for all $\alpha \in \mathscr{A}$ with $|\alpha|=n$ any $f \in F_{n}$ has constant value on the set

$$
\left\{\mu_{n+1} x_{\beta}+\left(1-\mu_{n+1}\right) z_{\beta}-x_{\alpha}: \beta \in S_{\alpha}\right\}
$$

and if we denote this value by $A_{\alpha}^{f}$ then $\left|A_{\alpha}^{f}\right| \leq a_{n} \cdot \varepsilon_{n}$.

We first take this construction for granted and use it to obtain the family $\left(w_{\alpha}\right)_{\alpha \in \mathscr{A}}$. This is done in the following way.

Set $u_{0}=x_{0}$ and for $\alpha \in \mathscr{A}$ with $|\alpha|=n$ and $\beta \in S_{\alpha}$ set

$$
u_{\beta}=\left(\prod_{k=1}^{n} \mu_{k}\right)\left(\mu_{n+1} x_{\beta}+\left(1-\mu_{n+1}\right) z_{\beta}-x_{\alpha}\right) \text {. }
$$

Let now $w_{a}=\sum_{\beta \leq \alpha} u_{\beta}$ for all $\alpha \in \mathscr{A}$. We claim that $\left(w_{\alpha}\right)_{\alpha \in \mathscr{A}}$ has the desired properties

(a) First of all we observe that each $w_{\alpha}$ is a convex combination of $x_{\alpha}$ and $\left\{z_{\beta}: \beta \leq \alpha\right\}$ and as such it belongs in $K$.

(b) Now we prove that $\left(w_{\alpha}\right)_{\alpha \in \mathscr{A}}$ is a $\frac{\delta}{8}$-approximate bush with bush function $\left(\lambda_{\alpha}\right)_{\alpha \in \mathscr{A}}$.

For $\alpha \in \mathscr{A}$ with $|\alpha|=n$ we have

$$
\begin{aligned}
& \left\|\sum_{\beta \in S_{\alpha}} \lambda_{\beta} w_{\beta}-w_{\alpha}\right\|=\left\|\sum_{\beta \in S_{\alpha}} \lambda_{\beta} u_{\beta}\right\| \\
& =\prod_{k=1}^{n} \mu_{k}\left\|\mu_{n+1} \sum_{\beta \in S_{\alpha}} \lambda_{\beta}\left(x_{\beta}-x_{\alpha}\right)+\left(1-\mu_{n+1}\right) \sum_{\beta \in S_{\alpha}} \lambda_{\beta}\left(z_{\beta}-x_{\alpha}\right)\right\| \\
& \leq\left\|\sum_{\beta \in S_{\alpha}} \lambda_{\beta} x_{\beta}-x_{\alpha}\right\|+\left(1-\mu_{n+1} \sum_{\beta \in S_{\alpha}} \lambda_{\beta}\left\|z_{\beta}-x_{\alpha}\right\|\right. \\
& \leq \frac{\delta}{2^{n+1} \cdot 50}+\frac{2 \delta}{2^{n+1} \cdot 100}=\frac{\delta}{2^{n} \cdot 50} .
\end{aligned}
$$


The last inequality is a consequence of property (ii), the inequality which occurs in property (vi) and the fact that $x_{\alpha}, z_{\beta} \in K \subset B_{x}$.

Furthermore for $\beta \in S_{\alpha}$ we get

$$
\begin{aligned}
\left\|w_{\beta}-w_{\alpha}\right\| & =\prod_{k=1}^{n} \mu_{k}\left\|\mu_{n+1}\left(x_{\beta}-x_{\alpha}\right)+\left(1-\mu_{n+1}\right)\left(z_{\beta}-x_{\alpha}\right)\right\| \\
& \geq \frac{1}{2}\left\|x_{\beta}-x_{\alpha}\right\|-\left(1-\mu_{n+1}\right)\left\|z_{\beta}-x_{\alpha}\right\| \\
& \geq \frac{\delta}{4}-\frac{\delta}{16} \cdot 2=\frac{\delta}{8} .
\end{aligned}
$$

We conclude that $\left(w_{\alpha}\right)_{\alpha \in \mathscr{A}}$ is indeed a $\frac{\delta}{8}$-approximate bush.

(c) To see that $\left(w_{\alpha}\right)_{\alpha \in \mathscr{A}}$ defines a C.F.D.S.D. we check that it satisfies the conditions of Lemma 1.5.

Indeed, it follows by (iv) that the $\left(w_{\alpha}\right)_{\alpha \in \mathscr{A}}$ are linearly independent.

By $(\mathrm{v})$ the set $F_{n}\left(1-\varepsilon_{n}\right)$-norms the linear span of $\left\{m_{\alpha}:|\alpha|=n\right\}$.

By (vi) for $f \in F_{n},|\alpha|=n, \beta \in S_{\alpha}, f\left(w_{\beta}-w_{\alpha}\right)=\prod_{k=1}^{n} \mu_{k} A_{\alpha}^{f}$ does not depend on $\beta$ and

$$
\begin{aligned}
\left|f\left(w_{\beta}-w_{\alpha}\right)\right| & =\prod_{k=1}^{n} \mu_{k}\left|A_{\alpha}^{f}\right| \leq \prod_{k=1}^{n} \mu_{k} a_{n} \varepsilon_{n} \\
& \leq \varepsilon_{n} \prod_{k=1}^{n} \mu_{k} \cdot \inf \left\{\left\|\sum_{|\alpha|=n} t_{\alpha} x_{\alpha}+y\right\|: \sum\left|t_{\alpha}\right|=1,\right. \\
\left.y \in\left\langle z_{\beta}:|\beta| \leq n\right\rangle\right\} & \\
& \leq \varepsilon_{n} \cdot \inf \left\{\left\|\sum_{|\alpha|=n} t_{\alpha} w_{\alpha}\right\|: \sum\left|t_{\alpha}\right|=1\right\} .
\end{aligned}
$$

So the hypotheses of Lemma 1.5. are fulfilled and the proof is complete.

We return to show that the initial construction is possible. We proceed by induction on the level of the tree $\mathscr{A}$.

0th level. Choose $0 \neq x_{0} \in D$ and $f_{0} \in B_{X^{*}}$ such that $f_{0}\left(x_{0}\right) \geq$ $\left(1-\varepsilon_{0}\right)\left\|x_{0}\right\|$.

Set $F_{0}=\left\{f_{0}\right\}$. 
$n+1$-st level. Suppose that the tree $A$ has been constructed up to the $n$th level and that $x_{\alpha} \in D, z_{\alpha} \in K, \lambda_{\alpha} \in R, \alpha \in \mathscr{A},|\alpha| \leq n$, $F_{k} \subset B_{X^{*}}, k \leq n, \mu_{k} \in R^{+}, k \leq n$ have been chosen to satisfy (i)-(vi).

Choose $\mu_{n+1}$ to satisfy

$$
0<1-\mu_{n+1} \leq \min \left\{\frac{a_{n} \varepsilon_{n}}{2}, 1-\rho_{n+1}\right\}
$$

where $a_{n}$ is as in (vi).

We first apply Lemma 1.6. to find a $\delta_{n+1}>0$, a finite-dimensional space $Y_{n+1}$ and numbers $A_{\alpha}^{f},|\alpha|=n, f \in F_{n}$ such that for any $\alpha \in \mathscr{A}$ with $|\alpha|=n$ and any $x \in K$ with $\left|f\left(x-x_{\alpha}\right)\right|<\delta_{n+1}$ for all $f \in F_{n}$, there exists a $z \in Y_{n+1} \cap K$ with

$$
f\left(\mu_{n+1} x+\left(1-\mu_{n+1}\right) z\right)=A_{\alpha}^{f}+f\left(x_{\alpha}\right) \text { for all } f \in F_{n} .
$$

Then

$$
\left|A_{\alpha}^{f}\right| \leq\left(1-\mu_{n+1}\right) \cdot 2=a_{n} \varepsilon_{n} .
$$

For each $\alpha \in \mathscr{A}$ with $|\alpha|=n$ consider the set

$$
D_{\alpha}=\left\{x \in D:\left\|x_{\alpha}-x\right\|>\frac{\delta}{2}\right\} \cap \mathscr{N}\left(x_{\alpha}, F_{n}, \delta_{n+1}\right)
$$

where

$$
\mathscr{N}\left(x_{\alpha}, F_{n}, \delta_{n+1}\right)=\left\{x \in X:\left|f\left(x-x_{\alpha}\right)\right|<\delta_{n+1} \text { for all } f \in F_{n}\right\} .
$$

By our choice of $D$ and $\delta, x_{\alpha}$ is a weak limit point of $D_{\alpha}$. So we can find $n \in N, x_{(\alpha, 1)}, \ldots, x_{\left(\alpha, n_{\alpha}\right)} \in D_{\alpha}$ and $\lambda_{(\alpha, 1)}, \ldots, \lambda_{\left(\alpha, n_{\alpha}\right)} \in R$ with $\sum_{i=1}^{n_{\alpha}} \lambda_{\alpha, i}=1$ such that

$$
\left\|\sum_{i=1}^{n_{\alpha}} \lambda_{\alpha, i} x_{\alpha, i}-x_{\alpha}\right\| \leq \frac{\delta}{2^{n+1} \cdot 50} .
$$

Since $D$ consists of linearly independent vectors and since the space

$$
Z_{n+1}=\left\langle\left\{x_{\beta}:|\beta| \leq n\right\} \cup\left\{z_{\beta}:|\beta| \leq n\right\} \cup Y_{n+1}\right\rangle
$$

is finite-dimensional, $x_{(\alpha, i)}, i=1, \ldots, n_{\alpha}$, can be taken to be linearly independent over $Z_{n+1}$.

Set now $S_{\alpha}=\left\{(\alpha, 1), \ldots,\left(\alpha, n_{\alpha}\right)\right\}$.

For $|\alpha|=n, \beta \in S_{\alpha}$ choose $z_{\beta} \in Y_{n+1} \cap K$ so that

$$
f\left(\mu_{n+1} x_{\beta}+\left(1-\mu_{n+1}\right) z_{\beta}\right)=A_{\alpha}^{f}+f\left(x_{\alpha}\right)
$$

for all $f \in F_{n}$. 
Finally choose $F_{n+1} \subset B_{X^{*}}\left(1-\varepsilon_{n+1}\right)$-norming the space $\left\langle\left\{x_{\alpha}:|\alpha| \leq\right.\right.$ $\left.n+1\} \cup\left\{z_{\beta}:|\beta| \leq n+1\right\}\right\rangle$.

This completes the inductive construction and the proof of the theorem.

It is known that the P.C.P. and the R.N.P. are not equivalent in general [B-R]. The second part of the present section is devoted to the investigation of the C.F.D.S.D. for non-dentable sets satisfying the additional property that the P.C.P. and the R.N.P. are equivalent on their subsets.

We recall first some definitions and known results.

2.4. Definition [G-G-M-S]. Let $\lambda$ be the Lebesgue measure on the interval $[0,1]$. A bounded linear operator $T: L^{1}(\lambda) \rightarrow X$ is said to be strongly regular if for all measurable subsets $A$ of $[0,1]$ with $\lambda(A)>0$ and every $\varepsilon>0$ there exists a weakly open subset $W$ of

$$
F_{A}=\left\{f: f \geq 0, \int f d \lambda=1, \operatorname{supp} f \subset A\right\}
$$

such that $\operatorname{diam} T[W]<\varepsilon$.

Next we state without proof a deep theorem. Its proof follows from the proof of Rosenthal [R] to Schachermayer's Theorem [S2]. For more information related to this we refer to $[\mathbf{A}-\mathbf{P}]$. We notice that a modification of the main result of $\S 3$ also gives a proof of this result.

2.5. TheOREM. Suppose that $K$ is a convex non-dentable subset of a B-space $X$ such that the R.N.P. is equivalent to the P.C.P. on its subsets. Then a bounded linear $K$-valued operator $T: L^{1}(\lambda) \rightarrow X$ is representable if and only if it is strongly regular. (Recall that $T$ is $K$-valued if it maps the densities of $L^{1}(\lambda)$ into $K$.)

Finally we will make use of the following result from [A-P] which is an "unconditional" version of the classical Mazur's Theorem.

2.6. LEMMA. Let $K$ be a bounded subset of the positive cone of $L^{1}(\lambda)$ and $f$ a weak limit point of $K$. Then for every $\varepsilon>0$ there exist a finite subset $F$ of $K, F=\left\{f_{1}, \ldots, f_{d}\right\}$ and positive reals $\left\{\alpha_{i}\right\}_{i=1}^{d}$, with $\sum_{i=1}^{d} \alpha_{i}=1$ such that

$$
\left\|\sum_{i=1}^{d} \sigma_{i} \alpha_{i}\left(f_{i}-f\right)\right\|<\varepsilon
$$

for all choices of signs $\sigma_{i}= \pm 1, i=1, \ldots, d$. 
We pass now to the statement and the proof of the second theorem of this section.

2.7. Theorem. Let $K$ be a closed convex non-dentable subset of a $B$-space $X$ and suppose that the P.C.P. and the R.N.P. are equivalent on its subsets. Then there exists a $\delta$-approximate bush $\left(w_{\alpha}\right)_{\alpha \in \mathscr{A}}$ defining a C.F.D.S.D. such that for all $\alpha \in \mathscr{A}$

$$
\left\|\sum_{\alpha \in S_{\alpha}} \sigma_{\beta} \lambda_{\beta}\left(w_{\beta}-w_{\alpha}\right)\right\|<\eta_{|\alpha|}
$$

for all choices of signs $\sigma_{\beta}= \pm 1, \sum_{n=0}^{\infty} \eta_{n}<\infty$ where $\left(\lambda_{\beta}\right)_{\beta \in \mathscr{A}}$ is the bush function.

2.8. REMARK. The additional property ensured by this theorem is that of the unconditional approximation of the elements $\left(w_{\alpha}\right)_{\alpha \in \mathscr{A}}$ by convex combinations of their successors. The role of this property will be discussed in $\S 4$. Here we can simply say that this property guarantees that the operator from $L^{1}(\lambda)$ induced by the averaged back bush is a Dunford-Pettis operator.

Proof of the Theorem. We construct the approximate bush $\left(w_{\alpha}\right)_{\alpha \in \mathscr{A}}$ following the same procedure as in the proof of Theorem 2.3. More precisely we produce an approximate bush $\left(x_{\alpha}\right)_{\alpha \in \mathscr{A}}$ and their "corrections" $\left(z_{\alpha}\right)_{\alpha \in \mathscr{A}} ;$ then the nodes $\left(u_{\alpha}\right)_{\alpha \in \mathscr{A}}$ of the desired bush $\left(w_{\alpha}\right)_{\alpha \in \mathscr{A}}$ are defined by the rule

$$
u_{\beta}=\left(\prod_{k=1}^{n} \mu_{k}\right)\left(\mu_{n+1}\left(x_{\beta}-x_{\alpha}\right)+\left(1-\mu_{n+1}\right)\left(z_{\beta}-x_{\alpha}\right)\right)
$$

where $|\alpha|=n, \beta \in S_{\alpha}$ and $\left(\mu_{n}\right)_{n \in N}$ are as in Theorem 2.3. Now

$$
\begin{gathered}
\left\|\sum_{\beta \in S_{\alpha}} \sigma_{\beta} \lambda_{\beta} u_{\beta}\right\| \leq\left\|\sum_{\beta \in S_{\alpha}} \sigma_{\beta} \lambda_{\beta}\left(x_{\beta}-x_{\alpha}\right)\right\|+2\left(1-\mu_{n+1}\right) \\
\leq\left\|\sum_{\beta \in S_{\alpha}} \sigma_{\beta} \lambda_{\beta}\left(x_{\beta}-x_{\alpha}\right)\right\|=2 \frac{\delta}{2^{|\alpha|} \cdot 100} .
\end{gathered}
$$

Hence to get the desired result it is enough to construct $\left(x_{\alpha}\right)_{\alpha \in \mathscr{A}}$ such 
that

$$
\left\|\sum_{\beta \in S_{\alpha}} \sigma_{\beta}\left(x_{\beta}-x_{\alpha}\right)\right\|<\frac{\delta}{2^{|\alpha|} \cdot 100} .
$$

Suppose now that $K$ is a subset of $B_{X}$ and consider a bounded linear nonrepresentable $K$-valued operator $T: L^{1}(\lambda) \rightarrow X$. Clearly $\|T\| \leq$ 1. By Theorem 2.5. $T$ is non-strongly regular; hence there exists a subset $A$ of $[0,1]$ with $\lambda(A)>0$ and $\delta>0$ such that diam $T[W]>$ $\delta$ for all weakly open subsets of $F_{A}$. As in Lemma 2.2 there exists an infinite subset of $T\left[F_{A}\right]$ consisting of linearly independent vectors.

Inductively we construct a $\frac{\delta}{2}$-approximate bush $\left(x_{\alpha}\right)_{\alpha \in \mathscr{A}}$ such that the inductive assumptions of Theorem 2.3 are fulfilled; further for each $\alpha, x_{\alpha}=T g_{\alpha}$ for some $g_{\alpha} \in F_{A}$ and

$$
\left\|\sum_{\beta \in S_{\alpha}} \sigma_{\beta} \lambda_{\beta}\left(x_{\beta}-x_{\alpha}\right)\right\|<\frac{\delta}{2^{|\alpha|} \cdot 100} .
$$

Suppose that $\left(x_{\alpha}\right)_{|\alpha| \leq n},\left(z_{\alpha}\right)_{|\alpha| \leq n}$ have been constructed. Choose $Y_{n}, \delta_{n}>0, F_{n}$ subset of $B_{X^{*}}$ as in Theorem 2.3 and for $\alpha \in \mathscr{A}$ with $|\alpha|=n$ we select a net $\left(f_{i}\right)_{i \in I}$ subset of $F_{A}$ weakly convergent to $g_{\alpha}$ with

$$
\left\|T g_{\alpha}-T f_{i}\right\| \geq \frac{\delta}{2}, \quad T^{*} x^{*}\left(f_{i}-g_{\alpha}\right) \leq \frac{\delta_{n}}{2}
$$

for all $x^{*} \in F_{n}$. Notice that the choice of $\left(f_{i}\right)_{i \in I}$ is possible because of the property of $F_{A}$. From Lemma 2.6 there exists a finite subset $\left\{f_{\beta}: \beta \in S_{\alpha}\right\}$ of $\left(f_{i}\right)_{i \in I}$ and $\left\{\lambda_{\beta}: \beta \in S_{\alpha}\right\}, \lambda_{\beta} \geq 0, \sum \lambda_{\beta}=1$ such that

$$
\left\|\sum_{\beta \in S_{\alpha}} \sigma_{\beta} \lambda_{\beta}\left(f_{\beta}-g_{\alpha}\right)\right\|<\frac{\delta}{2^{n+2} \cdot 100} .
$$

Next we choose a subset $\left\{h_{\beta}:|\beta|=n+1\right\}$ of $F_{A}$ such that $\left\{T h_{\beta}\right.$ : $|\beta|=n+1\}$ are linearly independent over the space generated by

$$
\left\{x_{\alpha}:|\alpha| \leq n\right\} \cup\left\{z_{\alpha}:|\alpha| \leq n\right\} \cup\left\{T f_{\beta}:|\beta|=n+1\right\} \cup Y_{n} .
$$

Finally we define

$$
g_{\beta}=l_{n+1} f_{\beta}+\left(1-l_{n+1}\right) h_{\beta}
$$

where $0<l_{n+1}<1$ are appropriately chosen so that

$$
\left\|x^{*}\left(T g_{\beta}-T g_{\alpha}\right)\right\|<\delta_{n}
$$


for $|\alpha|=n, \beta \in S_{\alpha}$ and $x^{*} \in F_{n}$ and

$$
\left\|\sum_{\beta \in S_{\alpha}} \sigma_{\beta} \lambda_{\beta}\left(g_{\beta}-g_{\alpha}\right)\right\|<\frac{\delta}{2^{n+1} \cdot 100} .
$$

We set $x_{\beta}=T g_{\beta}$ and continue the construction in the same manner as in the proof of Theorem 2.3.

This completes the proof of the theorem.

We pass now to the third part of this section. Here we deal with convex non-P.C.P. sets in Banach spaces with an unconditional basis and we will construct $\delta$-approximate bushes defining a C.F.D.S.D. and with the additional property that the nodes are well behaved with respect to the basis. Our construction is similar to a related construction given in [R-W]. First we introduce, for later use, a total order on a finitely branching tree.

2.9. Definition. Let $\mathscr{A}$ be a finitely branching tree. We denote by $\prec$ the following total order on $\mathscr{A}, \alpha \prec \beta$ iff either $|\alpha|<|\beta|$ or $|\alpha|=|\beta|$ and $\alpha$ is smaller than $\beta$ in the lexicographic order.

2.10. Definition. (a) Let $X$ be a Banach space with an unconditional basis $\left(e_{k}\right)_{k=1}^{\infty}$. A $\delta$-approximate bush $\left(x_{\alpha}\right)_{\alpha \in \mathscr{A}}$ is said to be unconditional if the nodes $\left(y_{\alpha}\right)_{\alpha \in \mathscr{A}}$ are linearly independent and for each $\alpha \in \mathscr{A}$ there exists a finite subset $M_{\alpha}$ of $N$ such that the sets $\left(M_{\alpha}\right)_{\alpha \in \mathscr{A}}$ are pairwise disjoint and if $\beta \in S_{\alpha}, y_{\beta}$ is supported by the set $\left(e_{k}\right)_{k \in M_{\alpha}}$ and $y_{0}$ is supported by $\left(e_{k}\right)_{k \in M_{-1}}$ where $M_{-1}$ is a finite set disjoint from each $M_{\alpha}$.

(b) A $\delta$-approximate bush $\left(x_{\alpha}\right)_{\alpha \in \mathscr{A}}$ is said to be almost unconditional if there exists an unconditional $\delta$-approximate bush $\left(w_{\alpha}\right)_{\alpha \in \mathscr{A}}$ such that

$$
\left\|y_{\alpha}-u_{\alpha}\right\|<\eta_{|\alpha|} \text { and } \sum_{k=0}^{\infty} \eta_{k}<\infty .
$$

Here $\left(y_{\alpha}\right)_{\alpha \in \mathscr{A}}$ and $\left(u_{\alpha}\right)_{\alpha \in \mathscr{A}}$ denote the nodes of the bushes $\left(x_{\alpha}\right)_{\alpha \in \mathscr{A}}$ and $\left(w_{\alpha}\right)_{\alpha \in \mathscr{A}}$ respectively.

2.11. Remark. (a) Suppose that $\left(x_{\alpha}\right)_{\alpha \in \mathscr{A}}$ is an unconditional $\delta$ approximate bush; then we easily verify that it defines a C.F.D.S.D. Furthermore if $x \in \overline{\mathrm{co}}\left(\tilde{x}_{\alpha}\right)_{\alpha \in \mathscr{A}}$

$$
x=\sum_{n=0}^{\infty} \sum_{|\alpha|=n} \lambda_{\alpha}^{(x)} y_{\alpha}
$$


then for all $\alpha \in \mathscr{A}$ the series

$$
\sum_{n=|\alpha||\beta|=n, \alpha<\beta}^{\infty} \sum_{\beta}^{(x)} y_{\beta}
$$

also converges in the norm; therefore if $\lambda_{\alpha}^{(x)} \neq 0$ the element

$$
x^{\alpha}=x_{\alpha}+\sum_{n=|\alpha|+1}^{\infty} \sum_{|\beta|=n, \alpha<\beta} \frac{\lambda_{\beta}^{(x)}}{\lambda_{\alpha}^{(x)}} y_{\beta}
$$

belongs to $\overline{\operatorname{co}}\left(\tilde{x}_{\alpha}\right)_{\alpha \in \mathscr{A}}$.

(b) Suppose that $\left(x_{\alpha}\right)_{\alpha \in \mathscr{A}}$ is an almost unconditional $\delta$-approximate bush defining a C.F.D.S.D. and $\left(w_{\alpha}\right)_{\alpha \in \mathscr{A}}$ the corresponding unconditional bush. Then if $\left(\mu_{\alpha}\right)_{\alpha \in \mathscr{A}}$ is a family of reals such that, for all $n, \sum_{|\alpha|=n}\left|\mu_{\alpha}\right|=1$, then the series

$$
\sum_{n=0}^{\infty} \sum_{|\alpha|=n} \mu_{\alpha} y_{\alpha}
$$

converges in norm if and only if

$$
\sum_{n=0}^{\infty} \sum_{|\alpha|=n} \mu_{\alpha} u_{\alpha}
$$

converges in norm.

Therefore if $K=\overline{\mathrm{co}}\left(\tilde{x}_{\alpha}\right)_{\alpha \in \mathscr{A}}$ and $L=\overline{\mathrm{co}}\left(\tilde{w}_{\alpha}\right)_{\alpha \in \mathscr{A}}$ the correspondence for $x \in K$,

$$
x=\sum_{n=0}^{\infty} \sum_{|\alpha|=n} \lambda_{\alpha}^{(x)} y_{\alpha}
$$

to

$$
\Phi(x)=\sum_{n=0}^{\infty} \sum_{|\alpha|=n} \lambda_{\alpha}^{(x)} u_{\alpha}
$$

defines a one-to-one and onto affine map.

2.12. REMARK. The previous observations show that for every almost unconditional $\delta$-approximate bush $\left(x_{\alpha}\right)_{\alpha \in \mathscr{A}}$ defining a C.F.D.S.D., if $x \in \overline{\operatorname{co}}\left(\tilde{x}_{\alpha}\right)_{\alpha \in \mathscr{A}}$, then for every $\alpha \in \mathscr{A}$ with $\lambda_{\alpha}^{(x)} \neq$ 0 the element $x^{\alpha}$ also belongs to $\overline{\mathrm{co}}\left(\tilde{x}_{\alpha}\right)_{\alpha \in \mathscr{A}}$. In [R-W] a $\delta$-bush $\left(x_{\alpha}\right)_{\alpha \in \mathscr{A}}$ with this property is said to be a strong martingale coordinatization. 
2.13. THEOREM. Let $X$ be a Banach space with an unconditional basis $\left(e_{k}\right)_{k=1}^{\infty}$. Then every closed convex subset $K$ of $X$ failing the $P$.C.P. contains an almost unconditional $\delta$-approximate bush defining a C.F.D.S.D.

Proof. Choose a sequence $\left(\eta_{n}\right)_{n=-1}^{\infty}$ of positive reals such that $\sum_{n=-1}^{\infty} \eta_{n}<\infty$. Inductively we shall construct:

A finitely branching tree $\mathscr{A}$; a family of intervals $\left\{\left[1, r_{-1}\right]\right\} \cup$ $\left\{\left[m_{\alpha}, r_{\alpha}\right]\right\}_{\alpha \in \mathscr{A}}$ of $N$ such that $r_{-1}<m_{\alpha}$ for all $\alpha \in \mathscr{A}$ and if $\alpha \prec \beta$ then $m_{\alpha} \leq r_{\alpha}<m_{\beta}$; two approximate bushes $\left(w_{\alpha}\right)_{\alpha \in \mathscr{A}},\left(v_{\alpha}\right)_{\alpha \in \mathscr{A}}$ sharing a common bush function and such that $\left(w_{\alpha}\right)_{\alpha \in \mathscr{A}}$ is contained in $K$ and defines a C.F.D.S.D. while the nodes $\left(u_{\alpha}\right)_{\alpha \in \mathscr{A}}$ of $\left(w_{\alpha}\right)_{\alpha \in \mathscr{A}}$ and the nodes $\left(d_{\alpha}\right)_{\alpha \in \mathscr{A}}$ of $\left(v_{\alpha}\right)_{\alpha \in \mathscr{A}}$ satisfy the following:

(i) $\left\|u_{0}-d_{0}\right\|<\eta_{-1}$ and for $\alpha \in \mathscr{A}, \beta \in S_{\alpha},\left\|u_{\beta}-d_{\beta}\right\|<\eta_{|\alpha|}$.

(ii) $d_{0} \in\left\langle e_{j}\right\rangle_{j=1}^{r-1}$ and for $\alpha \in \mathscr{A}, \beta \in S_{\alpha}, d_{\beta} \in\left\langle e_{j}\right\rangle_{j=m_{\alpha}}^{r_{\alpha}}$.

(iii) For $\alpha \in \mathscr{A}$ the vectors $\left\{d_{\beta}: \beta \in S_{\alpha}\right\}$ are linearly independent.

It is obvious that the approximate bush $\left(w_{\alpha}\right)_{\alpha \in \mathscr{A}}$ will satisfy the desired properties.

For the construction we repeat the procedure of the construction in the proof of Theorem 2.3 with the following modifications.

Let $D$ be as in the proof of Theorem 2.3. Denote by $P_{r}: X \rightarrow X$ the projection on $\left\langle e_{j}\right\rangle_{j=1}^{r}$. After choosing $x_{0}=y_{0} \in K \cap D$ chose $r_{-1} \in N$ such that $\left\|y_{0}-P_{r_{-1}}\left(y_{0}\right)\right\|<\frac{\eta_{-1}}{2}$ and set $d_{0}=P_{r_{-1}}\left(y_{0}\right)$.

Suppose now that for some $\alpha \in \mathscr{A}$ with $|\alpha|=n$ we have constructed the set

$$
\mathscr{A}_{\alpha}=\bigcup_{\substack{\gamma \in \mathscr{A} \\ \gamma \prec \alpha}} S_{\gamma}
$$

and that we have chosen $\left(x_{\gamma}\right)_{\gamma \in \mathscr{A}},\left(z_{\gamma}\right)_{\gamma \in \mathscr{A}}, F_{k}, \mu_{k}, k \leq n$, as in the proof of Theorem 2.3. Suppose in addition that we have chosen properly the integers $\left\{m_{\gamma}, r_{\gamma}\right\}_{\gamma \in \mathscr{A}}$. We wish to define the set $S_{\alpha}$, to choose the integers $\left\{m_{\alpha}, r_{\alpha}\right\}$ and to select $x_{\beta}$ and $d_{\beta}$ for all $\beta \in S_{\alpha}$.

Let $m_{\alpha}=\max \left\{r_{\gamma}: \gamma \in \mathscr{A}_{\alpha}\right\}+1$ and instead of the set $D_{\alpha}$ in the proof of Theorem 2.3 consider the set

$$
D_{\alpha}^{\prime}=D_{\alpha} \cap\left\{x:\left\|P_{m_{\alpha}-1}\left(x_{\alpha}-x\right)\right\| \leq \frac{\eta_{n}}{4}\right\}
$$

and choose $\left(x_{\beta}\right)_{\beta \in S_{\alpha}}$ as in that point, but contained in $D_{\alpha}^{\prime}$ and having the additional property of being linearly independent over $P_{m_{\alpha}-1}(X)$. 
For $\beta \in S_{\alpha}$ we set $y_{\beta}=x_{\beta}-x_{\alpha}$ and find $r_{\alpha} \geq m_{\alpha}$ such that

$$
\max _{\beta \in S_{\alpha}}\left\|y_{\beta}-P_{r_{\alpha}}\left(y_{\beta}\right)\right\| \leq \frac{\eta_{n}}{4}
$$

and the vectors

$$
\left\{\left(P_{r_{\alpha}}-P_{m_{\alpha}-1}\right)\left(y_{\beta}\right): \beta \in S_{\alpha}\right\}
$$

are linearly independent. Set

$$
d_{\beta}=\left(P_{r_{\alpha}}-P_{m_{\alpha}-1}\right)\left(y_{\beta}\right) .
$$

Then $\left\|y_{\beta}-d_{\beta}\right\|<\frac{\eta_{n}}{2}$ and if everything else is as in the original construction and $\mu_{n+1}$ is chosen to be close enough to 1 then $\left\|u_{\beta}-d_{\beta}\right\|<$ $\eta_{n}$.

This completes the inductive construction and the proof of the theorem.

3. The C.F.D.S.D. for non-dentable sets with the P.C.P. In this section we shall show that every closed convex bounded non-dentable set with the P.C.P. in a Banach space $X$ contains a $\delta$-approximate bush $\left(x_{\alpha}\right)_{\alpha \in \mathscr{A}}$ defining a C.F.D.S.D. Furthermore we shall show that the approximate bush can be chosen so that the norm and weak topologies coincide on the set $\overline{\operatorname{co}}\left(\tilde{x}_{\alpha}\right)_{\alpha \in \mathscr{A}}$.

We start with some known results necessary for the proof of the main theorem of this section.

3.1. Notation. Let $K$ be a closed convex bounded subset of a Banach space $X$. We denote by $\widetilde{K}$ the $w^{*}$ closure of $K$ in $X^{* *}$ and by Ext $\widetilde{K}$ the set of the extreme points of $\widetilde{K}$. A slice of the set $K$ is a set of the form

$$
T(K, f, \varepsilon)=\{x \in K: f(x)>\sup f \mid K-\varepsilon\}
$$

where $f \in X^{*}$ and $\varepsilon>0$. The slices will be denoted by the letters $S, T, R$.

If $G$ is a subset of $X$ and $x \in X$ we denote by $d(x, G)$ the distance of $x$ from $G$ and if $G_{1}, G_{2}$ are subsets of $X$ we denote by $d\left(G_{1}, G_{2}\right)$ the minimum distance of the elements of $G_{1}$ from the elements of $G_{2}$.

If $G$ is a subset of $X$ we denote by $\operatorname{diam}(G)$ the diameter of $G$ and if $f \in X^{*}$ we denote by $\operatorname{osc}(f \mid G)$ the oscillation of $f$ restricted on $G$. In the sequel $K$ always denotes a non-empty closed, convex, bounded subset of $X$.

The first lemma is due to Bourgain [B]. A proof of this can be found in $[\mathbf{R}]$ or $[\mathbf{S 1}]$. 
3.2. Lemma. Assume that $K$ is non-dentable. Let $G$ be a finite dimensional subspace, of $X^{* *}$ and $\varepsilon>0$. For any slices $S_{1}, \ldots, S_{n}$ of $K$, there exist $T_{k} \subset S_{k}, k=1, \ldots, n$, slices of $K$ and a finite subset $F$ of $B_{X^{*}}$ such that

$$
(1-\varepsilon)\left\|g+\sum_{k=1}^{n} \lambda_{k} y_{k}\right\| \leq \max _{f \in F} f\left(g+\sum_{k=1}^{n} \lambda_{k} y_{k}\right)
$$

for all $g \in G,\left(y_{k}\right)_{k=1}^{n} \in\left(T_{k}\right)_{k=1}^{n}$ and $\left(\lambda_{k}\right)_{k=1}^{n} \in R^{n}$.

3.3. Lemma. Suppose that $S$ is a slice of $K$ and $e \in \widetilde{S} \cap \operatorname{Ext} \widetilde{K}$. Then for every finite subset $F$ of $X^{*}$ and $\varepsilon>0$ there exists a slice $T$ contained in $S$ such that $e \in \widetilde{T}$ and $\operatorname{osc}(f \mid T)<\varepsilon$ for all $f \in F$.

(For a proof we refer to Lemma 2.1 of [S1].)

3.4. Lemma. Assume that $K$ is $2 \delta$-non-dentable, $S$ is a slice of $K$ and $\varepsilon>0$. Then there are slices $\left(S_{i}\right)_{i=1}^{n}$ with $\bar{S}_{i} \subset S$ and $\left(\lambda_{i}\right)_{i=1}^{n}$, $\lambda_{i}>0, \sum_{i=1}^{n} \lambda_{i}=1$ such that

$$
\operatorname{diam}\left(\sum_{i=1}^{n} \lambda_{i} S_{i}\right)<\varepsilon \text { and } d\left(\sum_{i=1}^{n} \lambda_{i} S_{i}, S_{j}\right)>\frac{\delta}{2}
$$

for any $j=1, \ldots, n$.

(For a proof see Lemma 2.9 of [S2].)

3.5. Lemma. Let $S_{1}, \ldots, S_{n}$ be slices of a $2 \delta$-non-dentable set $K$ and $G$ a finite dimensional subspace of $X$. Then there exist $T_{i} \subset S_{i}$, $i=1, \ldots, n$, slices of $K$ such that for $\left(x_{i}\right)_{i=1}^{n} \in\left(T_{i}\right)_{i=1}^{n}$ and $\left(\lambda_{i}\right)_{i=1}^{n}$

$$
\inf _{g \in G}\left\|g+\sum_{i=1}^{n} \lambda_{i} y_{i}\right\|>0
$$

Proof. Choose $e_{1} \in \widetilde{S}_{1} \cap \operatorname{Ext} \widetilde{K}$ with $d\left(e_{1}, G\right)>\frac{\delta}{2}$ ([R] Lemma 2.7). Set $G_{1}=\left\langle G \cup\left\{e_{1}\right\}\right\rangle$ and applying the same lemma find $e_{2} \in \widetilde{S}_{2} \cap \operatorname{Ext} \widetilde{K}$ with $d\left(e_{2}, G_{1}\right)>\frac{\delta}{2}$. Continuing in this manner we get a finite set $\left\{e_{1}, \ldots, e_{n}\right\} \subset \operatorname{Ext} \widetilde{K}$ with $e_{i} \in \widetilde{S}_{i}$ such that $d\left(e_{k}, G_{k-1}\right)>\frac{\delta}{2}$ where $G_{k-1}=\left\langle G \cup\left\{e_{1}, \ldots, e_{k-1}\right\}\right\rangle$ for $k=2, \ldots, n$.

It is clear that

$$
\inf \left\{\left\|g+\sum_{i=1}^{n} \lambda_{i} e_{i}\right\|: g \in G, \sum_{i=1}^{n}\left|\lambda_{i}\right|=1\right\}=\eta>0 .
$$


Choose now a finite $F$ subset of $B_{X^{*}}$ such that

$$
\left\|g+\sum_{i=1}^{n} \lambda_{i} e_{i}\right\| \leq 2 \max _{f \in F} f\left(g+\sum_{i=1}^{n} \lambda_{i} e_{i}\right),
$$

for any $g \in G,\left(\lambda_{i}\right)_{i=1}^{n} \in R^{n}$.

From Lemma 3.3 we find slices $T_{i} \subset S_{i}$ so that $e_{i} \in \widetilde{T}_{i}$ and $\operatorname{osc}\left(f \mid T_{i}\right)<\frac{\eta}{4}$ for all $f \in F$ and $i=1, \ldots, n$. It is easy to check now that for $\left(x_{i}\right)_{i=1}^{n} \in\left(T_{i}\right)_{i=1}^{n}$ and $\left(\lambda_{i}\right)_{i=1}^{n} \in R^{n}$

$$
\left\|g+\sum_{i=1}^{n} \lambda_{i} e_{i}\right\|>\frac{\eta}{4} \quad \text { for all } g \in G .
$$

The proof is complete.

The next result is due to Schachermayer ([S2] Lemma 2.8) see also ([R] Th. 2.2).

3.6. LEMMA. Let $K$ be non-dentable and $G$ a finite dimensional subspace of $X$. Then for any slices $S_{1}, \ldots, S_{n}$ of $K$ and $\varepsilon>0$ there exist $T_{i} \subset S_{i}, i=1, \ldots, n$, slices of $K$ such that for all $g \in G$ and $\left(\lambda_{i}\right)_{i=1}^{n}, \lambda_{i} \geq 0, \sum_{i=1}^{n} \lambda_{i}=1$

$$
d\left(g, \sum_{i=1}^{n} \lambda_{i} T_{i}\right) \geq \frac{1}{2} \operatorname{diam}\left(g, \sum_{i=1}^{n} \lambda_{i} T_{i}\right)-\varepsilon .
$$

We pass now to give the statement and the proof of the main theorem of this section.

3.7. Theorem. Let $K$ be a closed convex $\delta$-non-dentable subset of the ball of a separable Banach space $X$. If $K$ satisfies the Point of Continuity Property there exists a $\delta_{1}$-approximate bush $\left(w_{\alpha}\right)_{\alpha \in \mathscr{A}}$ defining a C.F.D.S.D. and such that on the set $W=\overline{\operatorname{co}}\left(\tilde{w}_{\alpha}\right)_{\alpha \in \mathscr{A}}$ the norm and weak topologies coincide.

3.8. REMARK. The part of the proof related to the C.F.D.S.D. strongly depends on Bourgain's proof of the well-known result that every Banach space with the P.C.P. failing the R.N.P. contains a subspace with a Finite-Dimensional Schauder Decomposition which fails the R.N.P. [B].

The fact that the norm and the weak topologies coincide on $W$ implies, as Rosenthal has proved in [R], that there is no extreme point in $W$. The existence of a non-dentable subset of $K$ without extreme 
points has been proved by Schachermayer [S2]. Our proof is in the spirit of Rosenthal's proof of Schachermayer's Theorem. The presence of the C.F.D.S.D. simplifies some arguments in the proof. The whole theorem can be considered as a unification of Bourgain's and Schachermayer's Theorems.

Proof of the Theorem. Fix an increasing sequence $\left(X_{n}\right)_{n=1}^{\infty}$ of finitedimensional subspaces of $X$ such that $X=\overline{\bigcup_{n \in N} X_{n}}$. Fix also $\left(\varepsilon_{n}\right)_{n=1}^{\infty},\left(\rho_{n}\right)_{n=1}^{\infty}$ sequences of positive reals with $\varepsilon_{n}<\frac{1}{2}, 1-\frac{\delta}{2^{n} \cdot 100} \leq$ $\rho_{n}<1$ and

$$
\prod_{n=1}^{\infty}\left(1-2 \varepsilon_{n}\right)>0, \quad \prod_{n=1}^{\infty} \rho_{n} \geq \frac{1}{2} .
$$

Inductively we construct the following: A finitely branching tree $\mathscr{A}$, a family $\left(T_{\alpha}\right)_{\alpha \in \mathscr{A}}$ of slices of $K$, two families of vectors of $K$, $\left(x_{\alpha}\right)_{\alpha \in \mathscr{A}}$ and $\left(z_{\alpha}\right)_{\alpha \in \mathscr{A}}$, a sequence $\left(F_{n}\right)_{n=0}^{\infty}$ of finite subsets of $B_{X^{*}}$, a sequence $\left(Y_{n}\right)_{n=0}^{\infty}$ of finite dimensional subspaces of $X$ and a sequence $\left(\mu_{n}\right)_{n=1}^{\infty}$ of positive reals satisfying the following properties:

(i) $x_{\alpha} \in T_{\alpha}$ and $T_{\beta} \subset T_{\alpha}$ for all $\alpha \in \mathscr{A}, \beta \in S_{\alpha}$.

(ii) $\lambda_{0}=1, \lambda_{\alpha}>0$ and $\sum_{\beta \in S_{\alpha}} \lambda_{\beta}=1$ for all $\alpha \in \mathscr{A}$.

(iii) If $|\alpha|=n$ then

$$
\begin{aligned}
& \operatorname{diam}\left(\sum_{\beta \in S_{\alpha}} \lambda_{\beta} T_{\beta}\right)<\frac{\delta}{2^{n+1} \cdot 100} \text { and } \\
& d\left(x_{\alpha}, \sum_{\beta \in S_{\alpha}} \lambda_{\beta} T_{\beta}\right)<\frac{\delta}{2^{n+1} \cdot 100} .
\end{aligned}
$$

(iv) $\left\|x_{\alpha}-x_{\beta}\right\|>\frac{\delta}{2}$ for all $\alpha \in \mathscr{A}, \beta \in S_{\alpha}$.

(v) $\left\{x_{\alpha}:|\alpha| \leq n\right\} \cup\left\{z_{\alpha}:|\alpha| \leq n+1\right\} \subset Y_{n} \cap K$.

(vi)

$$
\max _{f \in F_{n}} f\left(y+\sum_{|\alpha|=n} t_{\alpha} x_{\alpha}\right) \geq\left(1-\varepsilon_{n}\right)\left\|y+\sum_{|\alpha|=n} t_{\alpha} x_{\alpha}\right\|
$$

holds for all $y \in Y_{n-1}$ and reals $\left(t_{\alpha}\right)_{|\alpha|=n}$.

(vii) For $n \in N$

$$
a_{n}=\inf \left\{\left\|y+\sum_{|\alpha|=n} t_{\alpha} x_{\alpha}\right\|: y \in Y_{n-1}, \sum_{|\alpha|=n}\left|t_{\alpha}\right|=1\right\}
$$

is strictly positive. 
(viii) For $n \in N, \mu_{n}$ will be chosen so that

$$
\rho_{n} \leq \mu_{n}<1 \text { and } 1-\mu_{n+1} \leq \min \left\{\frac{a_{n} \cdot \varepsilon_{n}}{2}, \frac{\delta}{8}\right\}
$$

and for any $\alpha \in \mathscr{A},|\alpha|=n$ and $f \in F_{n}$ there exists a real $A_{\alpha}^{f}$ with $\left|A_{\alpha}^{f}\right| \leq a_{n} \cdot \varepsilon_{n}$ such that

$$
f\left(\mu_{n+1} x_{\beta}+\left(1-\mu_{n+1}\right) z_{\beta}\right)=A_{\alpha}^{f}+f\left(x_{\alpha}\right)
$$

for all $\beta \in S_{\alpha}$.

(ix)

$$
d\left(X_{n}, \sum_{|\alpha|=n} t_{\alpha} T_{\alpha}\right)>\frac{1}{2} \operatorname{diam}\left(\sum t_{\alpha} T_{\alpha}\right)-\frac{1}{n}
$$

holds for all $\left(t_{\alpha}\right)_{|\alpha|=n}, t_{\alpha} \geq 0, \sum_{|\alpha|=n} t_{\alpha}=1$.

We divide the rest of the proof into three stages. In the first, using the previous, we define the approximate bush $\left(w_{\alpha}\right)_{\alpha \in \mathscr{A}}$. The second is devoted to the inductive construction and in the third we will show that the norm and weak topologies coincide on $\overline{\operatorname{co}}\left(\tilde{w}_{\alpha}\right)_{|\alpha|=n}$.

Stage 1. The approximate busch $\left(w_{\alpha}\right)_{\alpha \in \mathscr{A}}$. First we notice that conditions (i), (ii), (iii) and (iv) imply that $\left(x_{\alpha}\right)_{\alpha \in \mathscr{A}}$ is a $\frac{\delta}{2}$-approximate bush with bush function $\left(\lambda_{\alpha}\right)_{\alpha \in \mathscr{A}}$.

We set now

$$
u_{0}=x_{0}
$$

and for all $n \in N, \alpha \in \mathscr{A},|\alpha|=n$ and $\beta \in S_{\alpha}$

$$
u_{\beta}=\left(\prod_{k=1}^{n} \mu_{k}\right)\left(\mu_{n+1} x_{\beta}+\left(1-\mu_{n+1}\right) z_{\beta}-x_{\alpha}\right) \text {. }
$$

Finally set

$$
w_{\alpha}=\sum_{\gamma \leq \alpha} u_{\gamma}
$$

We claim that $\left(w_{\alpha}\right)_{\alpha \in \mathscr{A}}$ is a $\frac{\delta}{8}$-approximate bush defining a C.F.D.S.D.

In fact the proof is identical with that of the same statement in the proof of Theorem 2.3 so we proceed to Stage 2 .

Stage 2. We show now how the inductive construction can be carried out. We proceed by induction: 
The 0-th level. Choose a slice $R_{0}$ of $K$ And $f_{0} \in B_{X^{*}}$ such that $f_{0}(x) \geq\left(1-\varepsilon_{0}\right)\|x\|$ for all $x \in R_{0}$.

Next choose a slice $R_{0}^{\prime}$ contained in $R_{0}$ with

$$
a_{0}=\inf _{x \in R_{0}}\|x\|>0 \text {. }
$$

Let $\mu_{1}$ be such that

$$
\rho_{1} \leq \mu_{1}<1 \text { and } 1-\mu_{1} \leq \min \left\{\frac{a_{0} \cdot \varepsilon_{0}}{2}, \frac{\delta}{8}\right\} .
$$

According to Lemma 1.6 there exists a finite dimensional subspace $Y_{0}^{\prime}$ of $X$ and $0<\delta_{0}$ so that for any $x \in K$ there exists a real $A_{x}^{f}$ such that if $y \in K$ and $\left|f_{0}(x-y)\right|<\delta_{0}$ there exists $z_{0} \in Y_{0}^{\prime} \cap K$ with

$$
f_{0}\left(\mu_{1} y+\left(1-\mu_{1}\right) z\right)=A_{x}^{f_{0}}+f_{0}(x) .
$$

An easy computation shows that $\left|A_{x}^{f_{0}}\right|<\alpha_{0} \cdot \varepsilon_{0}$.

Finally, from Lemma 3.3, we choose a slice $T_{0}$ with $T_{0} \subset R_{0}^{\prime}$ and $\operatorname{osc}\left(f \mid T_{0}\right)<\delta_{0}$. We set $F_{0}=\left\{f_{0}\right\}$.

The $n+1-$ st level. Assume that we have already built the tree up to the $n$th level and that we have chosen the slices $\left\{T_{\gamma}: \gamma \leq n\right\}$ and the sets $\left\{x_{\gamma}:|\gamma|<n\right\},\left\{z_{\gamma}:|\gamma|<n\right\}$, the subspaces $\left\{Y_{k}: k<n\right\}$ and the subsets $\left\{F_{k}: k \leq n\right\}$ of $B_{X^{*}}$ and the numbers $\left\{\mu_{k}: k \leq n+1\right\}$ so that conditions (i) to (ix) are satisfied. There exists a subspace $Y_{n}^{\prime}$ of $X$, and a $\delta_{n}>0$ satisfying the conclusion of Lemma 1.6. for the set of functionals $F_{n}$ and $\varepsilon=\mu_{n+1}$. It is easy to check that for $y \in K$ and $f \in F_{n}$ the number $A_{y}^{f}$ satisfies the inequality $\left|A_{y}^{f}\right|<\alpha_{n} \cdot \varepsilon_{n}$.

For each $\alpha$ with $|\alpha|=n$ we may choose (Lemma 3.4) slices $R_{(\alpha, 1)}, \ldots, R_{\left(\alpha, k_{\alpha}\right)}$ of $K$ and positive reals $\lambda_{(\alpha, 1)}, \ldots, \lambda_{\left(\alpha, k_{\alpha}\right)}$ with $\sum_{i=1}^{k_{\alpha}} \lambda_{(\alpha, i)}=1$ such that

$$
\begin{gathered}
R_{(\alpha, i)} \subset T_{\alpha} \text { for } i=1, \ldots, k_{\alpha}, \\
\operatorname{diam}\left(\sum_{i=1}^{k_{\alpha}} \lambda_{(\alpha, i)} R_{(\alpha, i)},\right)<\frac{\delta}{2^{n+1} \cdot 100}
\end{gathered}
$$

and

$$
d\left(\sum_{i=1}^{k_{\alpha}} \lambda_{(\alpha, i)} R_{(\alpha, i)}, R_{(\alpha, j)}\right)>\frac{\delta}{2} \quad \text { for } j=1, \ldots, k_{\alpha} ;
$$

we set $S_{\alpha}=\left\{(\alpha, 1), \ldots,\left(\alpha, k_{\alpha}\right)\right\}$. 
Choose $x_{\alpha} \in \sum_{\beta \in S_{\alpha}} \lambda_{\beta} R_{\beta}$ and $z_{\alpha} \in Y_{n}^{\prime}, A_{x_{\alpha}}^{f}, f \in F$ so that the conclusion of Lemma 1.6 is satisfied for $\varepsilon=1-\mu_{n}$.

By Lemma 3.2 we choose a finite subset $F_{n+1}$ of $B_{X^{*}}$ and $R_{\beta}^{\prime} \subset$ $R_{\beta},|\beta|=n+1$, slices of $K$ such that

$$
\max _{f \in F_{n+1}} f\left(y+\sum_{|\beta|=n+1} t_{\beta} y_{\beta}\right) \geq\left(1-\varepsilon_{n}\right)\left\|y+\sum_{|\beta|=n+1} t_{\beta} y_{\beta}\right\|
$$

holds for all $\left(y_{\beta}\right)_{|\beta|=n+1} \in\left(R_{\beta}^{\prime}\right)_{|\beta|=n+1},\left(t_{\beta}\right)_{|\beta|=n+1} \subset \mathbf{R}, y \in Y_{n}=$ $\left\langle Y_{n}^{\prime} \cup\left\{x_{\alpha}:|\alpha|=n\right\}\right\rangle$.

Next using Lemma 3.5 we find slices $R_{\beta}^{\prime \prime} \subset R_{\beta}^{\prime},|\beta|=n+1$, such that

$$
a_{n+1}:=\inf \left\{\left\|y+\sum_{|\beta|=n+1} t_{\beta} y_{\beta}\right\|: \sum_{|\beta|=n+1}\left|t_{\beta}\right|=1, y_{\beta} \in R_{\beta}^{\prime \prime}, y \in Y_{n}\right\}
$$

is strictly positive.

Choose $\mu_{n+2}$ real with $\rho_{n+2} \leq \mu_{n+2}<1$ and

$$
1-\mu_{n+2} \leq \min \left\{\frac{\alpha_{n+1} \cdot \varepsilon_{n+1}}{2}, \frac{\delta}{8}\right\} \text {. }
$$

Choose now $R_{\beta}^{\prime \prime \prime} \subset R_{\beta}^{\prime \prime},|\beta|=n+1$ slices of $K$ with $\operatorname{osc}\left(f \mid R_{\beta}^{\prime \prime \prime}\right)<$ $\delta_{n+1}$ for all $f \in F_{n+1}$ (Lemma 3.3).

Finally using Lemma 3.6 we choose $T_{\beta} \subset R_{\beta}^{\prime \prime \prime},|\beta|=n+1$, slices of $K$ such that

$$
d\left(X_{n}, \sum_{|\beta|=n+1} t_{\beta} T_{\beta}\right)>\frac{1}{2} \operatorname{diam}\left(\sum_{|\beta|=n+1} t_{\beta} T_{\beta}\right)-\frac{1}{n+1}
$$

for all $\left(t_{\beta}\right)_{|\beta|=n+1}, t_{\beta} \geq 0, \sum_{|\beta|=n+1} t_{\beta}=1$.

This completes the construction at the $(n+1)$-level and the proof of Stage 2.

Stage 3. The norm and weak topologies on $W=\overline{\mathrm{co}}\left(\tilde{w}_{\alpha}\right)_{\alpha \in \mathscr{A}}$ coincide.

We recall that $\left(\tilde{w}_{\alpha}\right)_{\alpha \in \mathscr{A}}$ is a $\frac{\delta}{8}$-approximate bush defining a C.F.D.S.D.; hence each element $x$ of $W$ has a unique representation of the form $\sum_{k=0}^{\infty} \sum_{|\alpha|=k} \lambda_{\alpha}^{(x)} u_{\alpha}$. We will use, for convenience, the following notation:

$$
M_{n}=\prod_{k=1}^{n} \mu_{k}, \quad M=\prod_{k=1}^{\infty} \mu_{k}, \quad \theta_{n}=M_{n}-M .
$$


Also we set $\mu_{0}=M_{0}=1$.

Notice that

$$
0<\theta_{n} \leq \sum_{k>n} 1-\mu_{k} \leq \frac{1}{2^{n}}
$$

therefore $\sum_{n=1}^{\infty} \theta_{n}<\infty$.

Fix an element $x$ of $W$ with representation

$$
x=\sum_{k=0}^{\infty} \sum_{|\alpha|=k} \lambda_{\alpha}^{(x)} u_{\alpha}
$$

and $\varepsilon>0$. Then

$$
\begin{aligned}
\sum_{k=0}^{\infty} \sum_{|\alpha|=k} \lambda_{\alpha}^{(x)} u_{\alpha}= & \sum_{k=0}^{\infty} M_{k} \sum_{|\alpha|=k} \lambda_{\alpha}^{(x)} y_{\alpha} \\
& +\sum_{k=0}^{\infty} M_{k-1}\left(1-\mu_{k}\right) \sum_{|\alpha|=k} \lambda_{\alpha}^{(x)} v_{\alpha}
\end{aligned}
$$

where $v_{\alpha}=z_{\alpha}-x_{\left.\alpha\right|_{|\alpha|-1}}$. The left side of the above equality converges in the norm of $X$ also the second series of the right side converges absolutely. Now writing

$$
\sum_{k=0}^{\infty} M_{k} \sum_{|\alpha|=k} \lambda_{\alpha}^{(x)} y_{\alpha}=M \cdot \sum_{k=0}^{\infty} \sum_{|\alpha|=k} \lambda_{\alpha}^{(x)} y_{\alpha}+\sum_{k=0}^{\infty} \theta_{k} \sum_{|\alpha|=k} \lambda_{\alpha}^{(x)} y_{\alpha}
$$

and using (1) we get that $\sum_{k=0}^{\infty} \sum_{|\alpha|=k} \lambda_{\alpha}^{(x)} y_{\alpha}$ converges in the norm of $X$.

Recall that $\left(y_{\alpha}\right)_{\alpha \in \mathscr{A}}$ denote the nodes of $\left(x_{\alpha}\right)_{\alpha \in \mathscr{A}}$.

Choose $N_{0}$ such that for all $n>N_{0}$

$$
d\left(\sum_{k=0}^{n} \sum_{|\alpha|=k} \lambda_{\alpha}^{(x)} y_{\alpha}, X_{n}\right)<\frac{\varepsilon}{2}
$$

Since

$$
\sum_{k=0}^{n} \sum_{|\alpha|=n} \lambda_{\alpha}^{(x)} y_{\alpha} \in \sum_{|\alpha|=n} \lambda_{\alpha}^{(x)} T_{\alpha}
$$

we get that for $n>\max \left(N_{0}, \frac{1}{\varepsilon}\right)$

$$
\operatorname{diam}\left(\sum_{|\alpha|=n} \lambda_{\alpha}^{(x)} T_{\alpha}\right)<2 \varepsilon
$$

(Condition (ix).) 
Choose $N_{1}$ such that

$$
\operatorname{diam}\left(\sum_{|\alpha|=N_{1}} \lambda_{\alpha}^{(x)} T_{\alpha}\right)<2 \varepsilon
$$

and

$$
\frac{1}{2 N_{1}}<\frac{\varepsilon}{4}
$$

Notice also that (1) and (3) imply that

$$
\sum_{k>N_{1}} \theta_{k}<\frac{\varepsilon}{4}
$$

We recall that the coefficient functionals $\left(f_{\alpha}\right)_{\alpha \in \mathscr{A}}$ are weakly continuous on $W$; hence the following set

$$
W(x)=\left\{y \in W: \sum_{n=0}^{N_{1}} \sum_{|\alpha|=n}\left|\lambda_{\alpha}^{(y)}-\lambda_{\alpha}^{(x)}\right|<\frac{\varepsilon}{4}\right\}
$$

defines a weak neighborhood of $x$ into the set $W$.

Claim. $W(x) \subset B(x, 7 \varepsilon)$.

Choose $y \in W(x)$; then

$$
\begin{aligned}
x-y= & \sum_{n=0}^{\infty} \sum_{|\alpha|=n}\left(\lambda_{\alpha}^{(x)}-\lambda_{\alpha}^{(y)}\right) u_{\alpha}=\sum_{n=0}^{\infty} M_{n} \sum_{|\alpha|=n}\left(\lambda_{\alpha}^{(x)}-\lambda_{\alpha}^{(y)}\right) y_{\alpha} \\
& +\sum_{n=1}^{\infty} M_{n}\left(1-\mu_{n}\right) \sum_{|\alpha|=n}\left(\lambda_{\alpha}^{(x)}-\lambda_{\alpha}^{(y)}\right) v_{\alpha} .
\end{aligned}
$$

We first treat the second series of the right side.

$$
\begin{aligned}
& \left\|\sum_{n=1}^{\infty} M_{n}\left(1-\mu_{n}\right) \sum_{|\alpha|=n}\left(\lambda_{\alpha}^{(x)}-\lambda_{\alpha}^{(y)}\right) v_{\alpha}\right\| \\
& \leq\left\|\sum_{n=1}^{N_{1}} M_{n}\left(1-\mu_{n}\right) \sum_{|\alpha|=n}\left(\lambda_{\alpha}^{(x)}-\lambda_{\alpha}^{(y)}\right) v_{\alpha}\right\| \\
& \quad+\left\|\sum_{n>N_{1}} M_{n}\left(1-\mu_{n}\right) \sum_{|\alpha|=n}\left(\lambda_{\alpha}^{(x)}-\lambda_{\alpha}^{(y)}\right) v_{\alpha}\right\| \\
& <\varepsilon+4 \cdot\left(\sum_{n>N_{1}} M_{n}\left(1-\mu_{n}\right)\right)<2 \varepsilon .
\end{aligned}
$$


We estimate now the norm of the first series of the right side.

$$
\begin{aligned}
& \sum_{n=0}^{\infty} M_{n} \sum_{|\alpha|=n}\left(\lambda_{\alpha}^{(x)}-\lambda_{\alpha}^{(y)}\right) y_{\alpha} \\
& \quad=M \cdot \sum_{n=0}^{\infty} \sum_{|\alpha|=n}\left(\lambda_{\alpha}^{(x)}-\lambda_{\alpha}^{(y)}\right) y_{\alpha}+\sum_{n=0}^{\infty} \theta_{n} \sum_{|\alpha|=n}\left(\lambda_{\alpha}^{(x)}-\lambda_{\alpha}^{(y)}\right) y_{\alpha} .
\end{aligned}
$$

Since $\sum_{n>N_{1}} \theta_{n}<1 / 2^{N_{1}}<\varepsilon / 4$ we get

$$
\begin{aligned}
& \left\|\sum_{n=0}^{\infty} \theta_{n} \sum_{|\alpha|=n}\left(\lambda_{\alpha}^{(x)}-\lambda_{\alpha}^{(y)}\right) y_{\alpha}\right\| \\
& \quad \leq\left\|\sum_{n=0}^{N_{1}} \theta_{n} \sum_{|\alpha|=n}\left(\lambda_{\alpha}^{(x)}-\lambda_{\alpha}^{(y)}\right) y_{\alpha}\right\|+\left\|\sum_{n>N_{1}} \theta_{n} \sum_{|\alpha|=n}\left(\lambda_{\alpha}^{(x)}-\lambda_{\alpha}^{(y)}\right) y_{\alpha}\right\| \\
& \quad<2 \cdot \frac{\varepsilon}{4}+4 \cdot \frac{\varepsilon}{4}<2 \varepsilon .
\end{aligned}
$$

We finally estimate

$$
M \cdot\left\|\sum_{n=0}^{\infty} \sum_{|\alpha|=n}\left(\lambda_{\alpha}^{(x)}-\lambda_{\alpha}^{(y)}\right) y_{\alpha}\right\|
$$

and since $M<1$ it is sufficient to estimate only the norm of the vector.

Recall that

$$
\sum_{n=0}^{\infty} \sum_{|\alpha|=n} \lambda_{\alpha}^{(x)} y_{\alpha} \in \overline{\sum_{|\alpha|=N_{1}} \lambda_{\alpha}^{(x)} T_{\alpha}}\|\cdot\|
$$

and

$$
\sum_{n=0}^{\infty} \sum_{|\alpha|=n} \lambda_{\alpha}^{(x)} y_{\alpha} \in \overline{\sum_{|\alpha|=N_{1}} \lambda_{\alpha}^{(y)} T_{\alpha}}\|\cdot\|
$$

By the definition of $W(x)$ and by (2) for $h_{\alpha}, g_{\alpha} \in T_{\alpha},|\alpha|=N_{1}$ we get

$$
\begin{aligned}
& \left\|\sum_{|\alpha|=N_{1}} \lambda_{\alpha}^{(x)} h_{\alpha}-\sum_{|\alpha|=N_{1}} \lambda_{\alpha}^{(y)} g_{\alpha}\right\| \\
& \leq\left\|\sum_{|\alpha|=N_{1}}\left(\lambda_{\alpha}^{(x)}-\lambda_{\alpha}^{(y)}\right) g_{\alpha}\right\|+\left\|\sum_{|\alpha|=N_{1}} \lambda_{\alpha}^{(x)} g_{\alpha}-\sum_{|\alpha|=N_{1}} \lambda_{\alpha}^{(x)} h_{\alpha}\right\| \\
& \quad<\frac{\varepsilon}{2}+2 \varepsilon<3 \varepsilon .
\end{aligned}
$$


In particular

$$
\left\|\sum_{n=0}^{\infty} \sum_{|\alpha|=n}\left(\lambda_{\alpha}^{(x)}-\lambda_{\alpha}^{(y)}\right) y_{\alpha}\right\|<3 \varepsilon .
$$

From $\left(B_{1}\right)$ and $\left(B_{2}\right)$ we get

$$
\left\|\sum_{n=0}^{\infty} M_{n} \sum_{|\alpha|=n}\left(\lambda_{\alpha}^{(x)}-\lambda_{\alpha}^{(y)}\right) y_{\alpha}\right\|<5 \varepsilon
$$

(A) and (B) imply that

$$
\|x-y\|<7 \varepsilon .
$$

This completes the proof of the theorem.

4. $\mathscr{P} a l$-representation for certain convex sets.

4.1. Definition. We denote by $\mathscr{P} a l$ the set of all probability atomless (diffuse) measures on $[0,1]$. A closed convex set $K$ in a Banach space $X$ has a $\mathscr{P} a l$-representation if there exists $T: \mathscr{P}_{a} \rightarrow$ $K$ affine, one to one, onto and continuous.

4.2. REMARK. $\mathscr{P} a l$ is a norm-closed convex subset of $M[0,1]$ without extreme points. Hence if $K$ has a $\mathscr{P} a l$-representation then $\operatorname{Ext}[K]=\varnothing$.

The problem of whether or not, every closed convex $K$ failing R.N.P. contains a closed subset $L$ with a $\mathscr{P} a l$-representation was brought to the attention of the authors by Professor Rosenthal. Our goal is to establish $\mathscr{P} a$-representations for certain classes of nondentable sets.

4.3. REMARK. If $S$ is an uncountable compact metric space we denote by $\mathscr{P}_{a} \ell(S)$ the probability atomless measures on $S$. It is known that for any such $S$ the space $M(S)$ is lattice isometric to the space

$$
\left(l^{1}(S) \oplus \sum_{\xi<2^{\omega}} \oplus L^{1}\left(\mu_{\xi}\right)\right)_{1}
$$

where $L^{1}\left(\mu_{\xi}\right)$ is lattice isometric to $L^{1}[0,1]$.

Hence $\mathscr{P}_{a}(S)$ is isometric to the densities of the space

$$
\left(\sum_{\xi<2^{\omega}} \oplus L^{1}\left(\mu_{\xi}\right)\right)_{1}
$$


Furthermore if we denote by $D\left(\mu_{\xi}\right)$ the densities of $L^{1}\left(\mu_{\xi}\right)$ then $\mathscr{P} a \ell(S)$ is affinely isometric to the set

$$
\overline{\mathrm{co}}\left[\bigcup_{\xi<2^{\omega}} D\left(\mu_{\xi}\right)\right] .
$$

From all the above we get that for every uncountable set $S$, $\mathscr{P}_{a}(S)$ is affinely isometric to $\mathscr{P} a l$ and if $\left(\mu_{\gamma}\right)_{\gamma \in \Gamma}$ is a family of mutually singular probability atomless measures on $S$ then

$$
\overline{\mathrm{co}}\left(\bigcup_{\gamma \in \Gamma} D\left(\mu_{\gamma}\right)\right)_{\gamma \in \Gamma}
$$

is affinely isometric to $\mathscr{P} a \ell$ if and only if $|\Gamma|=2^{\omega}$.

4.4. Notations-Remarks. Let $\mathscr{A}$ be a finitely branching tree. We denote by $S$ the set of all infinite branches corresponding to $\mathscr{A}$. That is,

$$
S=\left\{s \in N^{N}: \forall n \in N, s \mid n \in \mathscr{A}\right\} .
$$

We topologize $S$ by defining a basis of open sets

$$
V_{\alpha}=\{s \in S: s|| \alpha \mid=\alpha\} \quad \text { for all } \alpha \in \mathscr{A} .
$$

Each $V_{\alpha}$ is a clopen set and $S$ with this topology is a compact metric space.

Suppose next that $\left(x_{\alpha}\right)_{\alpha \in \mathscr{A}}$ is a bounded $\delta$-approximate bush, $\left(\lambda_{\alpha}\right)_{\alpha \in \mathscr{A}}, \lambda_{\alpha} \neq 0$ the corresponding bush function and $K=\overline{\mathrm{co}}\left(\tilde{x}_{\alpha}\right)_{\alpha \in \mathscr{A}}$. We define a probability measure $\lambda$ on $S$ by the rule

$$
\lambda\left(V_{\alpha}\right)=\prod_{\beta \leq \alpha} \lambda_{\beta} .
$$

So $\lambda$ is strictly positive atomless.

Further we define

$$
T\left(\chi_{V_{\alpha}}\right)=\lambda\left(V_{\alpha}\right) \cdot \tilde{x}_{\alpha}
$$

and there exists a unique extension of $T$ to a linear bounded $K$-valued operator $T: L^{1}(S, \lambda) \rightarrow X$.

Finally setting $\varphi_{n}: S \rightarrow X$ to be the function defined by the rule

$$
\varphi_{n}(s)=\tilde{x}_{\alpha} \quad \text { iff }|\alpha|=n, s \in V_{\alpha}
$$

then the sequence $\left(\varphi_{n}\right)_{n \in N}$ defines a martingale corresponding to the operator $T$. 
Consider now a $\delta$-approximate bush $\left(x_{\alpha}\right)_{\alpha \in \mathscr{A}}$ defining a C.F.D.S.D. and let $K=\overline{\mathrm{co}}\left(\tilde{x}_{\alpha}\right)_{\alpha \in \mathscr{A}}$.

We have already proved (Prop. 1.3.) that each $x$ in $K$ has a unique representation of the form

$$
x=\sum_{n=0}^{\infty} \sum_{|\alpha|=n} \lambda_{\alpha}^{(x)} y_{\alpha}
$$

where $\lambda_{0}^{(x)}=1$ and for all $\alpha \in \mathscr{A}, \lambda_{\alpha}^{(x)}=\sum_{\beta \in S_{\alpha}} \lambda_{\beta}^{(x)}$.

Therefore for every $x \in K$ there exists a unique probability measure $\mu_{x}$ on $S$ defined by the rule $\mu_{x}\left(V_{\alpha}\right)=\lambda_{\alpha}^{(x)}$.

We set

$$
M_{K}=\left\{\mu \in M_{1}^{+}(S): \exists x \in K, \quad \mu=\mu_{x}\right\} .
$$

The set $M_{K}$ is a convex norm-closed subset of $M(S)$ and the map

$$
T^{*}: M_{K} \rightarrow K
$$

such that $T^{*}\left(\mu_{x}\right)=x$ is an affine and norm continuous function.

4.5. Definition. Let $\left(x_{\alpha}\right)_{\alpha \in \mathscr{A}}$ be a $\delta$-approximate bush defining a C.F.D.S.D. and $K, M_{K}$ as before. The approximate bush $\left(x_{\alpha}\right)_{\alpha \in \mathscr{A}}$ is said to be adequate if for every $\mu \in M_{K}, D(\mu)$ is also a subset of $M_{K}$.

4.6. REMARK. In [R-W] the notion of the strong martingale coordinatization is considered. This is formally weaker than our adequate bush notion, but it turns out that they are equivalent. Indeed, using our terminology the strong martingale coordinatization means that for all $\mu \in M_{K}$ and $\alpha \in \mathscr{A}$ such that $\mu\left(V_{\alpha}\right) \neq 0$ the measure

$$
\mu_{\alpha}=\frac{\mu \mid V_{\alpha}}{\mu\left(V_{\alpha}\right)}
$$

also belongs to $M_{K}$. But we easily verify that $D(\mu)=\overline{c o}\left(\mu_{\alpha}\right)_{\alpha \in \mathscr{A}}$; hence the strong martingale coordinatization implies that the approximate bush is adequate.

4.7. LEMMA. If $\left(x_{\alpha}\right)_{\alpha \in \mathscr{A}}$ is a $\delta$-approximate adequate bush defining a C.F.D.S.D. then

(i) Every $\mu \in M_{K}$ is atomless.

(ii) There exists a family $\left(\mu_{\gamma}\right)_{\gamma \in \Gamma}$ of pairwise singular elements of $M_{K}$ such that

$$
M_{K}=\overline{\operatorname{co}}\left(\bigcup_{\gamma \in \Gamma} D\left(\mu_{\gamma}\right)\right) \text {. }
$$


Proof. (i) To see that each measure in $M_{K}$ is atomless we notice that if $\delta_{s}$ is an atom for such a measure $\mu$ then $\delta_{s}$ also belongs to $M_{K}$. But then

$$
T^{*}\left(\delta_{s}\right)=\sum_{n=0}^{\infty} y_{s \mid n}
$$

and the series diverges, a contradiction.

(ii) Using Zorn's lemma we choose a maximal family of pairwise singular measures $\left\{\mu_{\gamma}: \gamma \in \Gamma\right\}$ of elements of $M_{K}$. Clearly the set $\overline{\mathrm{co}}\left(\bigcup_{\gamma \in \Gamma} D\left(\mu_{\gamma}\right)\right)$ is a subset of $M_{K}$. If $\mu$ belongs to the difference of the two sets then $\mu=l \nu_{1}+(1-l) \nu_{2}$ where $\nu_{1}, \nu_{2} \in M_{1}^{+}(S)$, $\nu_{1} \in \overline{\mathrm{co}}\left(\bigcup_{\gamma \in \Gamma} D\left(\mu_{\gamma}\right)\right)$ and $\nu_{2}$ is singular to each $\mu_{\gamma}$. To see that $\nu_{2} \in M_{K}$ we notice that the series

$$
\sum_{n=0}^{\infty} \sum_{|\alpha|=n} \nu_{2}\left(V_{\alpha}\right) \cdot x_{\alpha}
$$

converges in norm since

$$
\nu_{2}\left(V_{\alpha}\right)=\frac{\mu\left(V_{\alpha}\right)-l \nu_{1}\left(V_{\alpha}\right)}{1-l}
$$

and the corresponding series with coefficients $\left(\mu\left(V_{\alpha}\right)\right)_{\alpha \in \mathscr{A}}$, $\left(\nu_{1}\left(V_{\alpha}\right)\right)_{\alpha \in \mathscr{A}}$ converge in norm. This contradiction completes the proof.

4.8. Proposition. Let $X$ be a Banach space and let $\left(x_{\alpha}\right)_{\alpha \in \mathscr{A}}$ be a $\delta$-approximate bush defining a C.F.D.S.D. Then in each of the following cases the bush is adequate.

(i) On the set $K=\overline{\mathrm{CO}}\left(\tilde{x}_{\alpha}\right)_{\alpha \in \mathscr{A}}$ norm and weak topologies coincide.

(ii) The space $X$ has an unconditional basis and $\left(x_{\alpha}\right)_{\alpha \in \mathscr{A}}$ is almost unconditional.

(iii) The set $\left(x_{\alpha}\right)_{\alpha \in \mathscr{A}}$ is a subset of the positive cone of $L^{1}[0,1]$.

Proof. (i) Let $\mu$ be an element of $M_{K}$ and for $\alpha \in \mathscr{A}$ with $\mu\left(V_{\alpha}\right) \neq$ 0 we set

$$
\mu_{\alpha}=\frac{\mu \mid V_{\alpha}}{\mu\left(V_{\alpha}\right)} .
$$

Then for all $n$ we have

$$
\mu=\sum_{|\alpha|=n} \mu\left(V_{\alpha}\right) \cdot \mu_{\alpha} .
$$


Consider $X$ as a subspace of $Y^{*}$ with $Y$ separable and let $\widetilde{K}$ be the $w^{*}$-closure of $K$ in $Y^{*}$. For each $\alpha,|\alpha|=n$, consider the sequence $\left(x_{\alpha}^{m}\right)_{m=1}^{\infty}$ where

$$
x_{\alpha}^{m}=\sum_{|\beta|=m} \mu_{\alpha}\left(V_{\beta}\right) \cdot x_{\beta}
$$

and let $M \subset N$ be an infinite set such that for all $\alpha$ with $|\alpha|=n$ the sequence $\left(x_{\alpha}^{m}\right)_{m \in M} w^{*}$-converges to some $w_{\alpha}$ in $\widetilde{K}$. Then clearly we have that

$$
T^{*}(\mu)=\sum_{|\alpha|=n} \mu\left(V_{\alpha}\right) \cdot w_{\alpha} .
$$

Since $T^{*}(\mu)$ is a point of continuity it follows that the same property holds for each $w_{\alpha}$ and hence each $w_{\alpha}$ belongs to $K$ ([L-L-T] and [R]). Now we easily check that $\mu_{\alpha}=\mu_{w_{\alpha}}$ and hence $\mu_{\alpha} \in M_{K}$.

Therefore the approximate bush $\left(x_{\alpha}^{\alpha}\right)_{\alpha \in \mathscr{A}}$ is a strong martingale coordinatization which as we mentioned before implies that $\left(x_{\alpha}\right)_{\alpha \in \mathscr{A}}$ is adequate.

(ii) If $K=\overline{\operatorname{co}}\left(\tilde{x}_{\alpha}\right)_{\alpha \in \mathscr{A}}$ and $\mu \in M_{K}, \alpha \in \mathscr{A}$ with $\mu\left(V_{\alpha}\right) \neq 0$ Remark 2.12 implies that $\mu_{\alpha} \in M_{k}$ and hence $\left(x_{\alpha}\right)_{\alpha \in \mathscr{A}}$ is adequate.

(iii) Again we set $K=\overline{\operatorname{co}}\left(\tilde{x}_{\alpha}\right)_{\alpha \in \mathscr{A}}$ and we denote by $\widetilde{K}$ the $w^{*}$ closure of $K$ in $\left(L^{1}[0,1]\right)^{* *}$. Choose $\mu \in M_{K}$ and for fixed $n$ write

$$
\mu=\sum_{|\alpha|=n} \mu\left(V_{\alpha}\right) \cdot \mu_{\alpha}
$$

Consider the sequence $\left(x_{\alpha}^{m}\right)_{m=n}^{\infty}$, where

$$
x_{\alpha}^{m}=\sum_{|\beta|=m} \mu_{\alpha}\left(V_{\beta}\right) \cdot x_{\beta} .
$$

There exists a subnet $\left(x_{\alpha}^{m_{J}}\right)_{j \in J}$ of $\left(x_{\alpha}^{m}\right)_{m=n}^{\infty}$ such that for all $\alpha$ with $|\alpha|=n,\left(x_{\alpha}^{m_{J}}\right)_{j \in J} w^{*}$-converges to some $w_{\alpha} \in \widetilde{K}$. Each $w_{\alpha}$ belongs to the positive cone of $\left(L^{1}[0,1]\right)^{* *}$ and

$$
T^{*}(\mu)=\sum_{|\alpha|=n} \mu\left(V_{\alpha}\right) \cdot w_{\alpha}
$$

so $\mu\left(V_{\alpha}\right) \cdot w_{\alpha}<T^{*}(\mu)$, where " $<$ " denotes the order in the lattice $\left(L^{1}[0,1]\right)^{* *}$. Since $L^{1}[0,1]$ is a solid Banach lattice, $\mu\left(V_{\alpha}\right) \cdot w_{\alpha} \in$ $L^{1}[0,1]$ and we conclude that $w_{\alpha} \in \widetilde{K} \cap L^{1}[0,1]=K$. It is clear now that $\mu_{w_{\alpha}}=\mu_{\alpha}$. 
4.9. Lemma. Suppose that $\left(x_{\alpha}\right)_{\alpha \in \mathscr{A}}$ is an adequate bush, $K=$ $\overline{\mathrm{co}}\left(\tilde{x}_{\alpha}\right)_{\alpha \in \mathscr{A}}$ and $M_{K}$ is non-separable. Then $M_{K}$ is isometric to $\mathscr{P} a l$.

Proof. We have shown (Lemma 4.7) that $M_{K}$ has the form $\overline{\mathrm{co}}\left(\bigcup_{\gamma \in \Gamma} D\left(\mu_{\gamma}\right)\right)$ where $\left(\mu_{\gamma}\right)_{\gamma \in \Gamma}$ is a family of pairwise singular probability measures on $S$. In order to show that $M_{K}$ is isometric to $\mathscr{P}$ al it is enough to prove that $|\Gamma|=2^{\omega}$. Since for each $\mu$ in $M(S) L^{1}(\mu)$ is separable we will get the desired result provided that we show that the norm density character of $M_{K}$ is $2^{\omega}$. Hence we prove the next

Claim. The density character $d\left(M_{K}\right)$ of $M_{K}$ is $2^{\omega}$.

Clearly $d\left(M_{K}\right) \leq 2^{\omega}$. Therefore we need to show that $d\left(M_{K}\right) \geq 2^{\omega}$ which follows if we prove that there exists a subset $D$ of $M_{K}$ with $d(D)=2^{\omega}$.

We set $\mathscr{D}$ the dyadic tree i.e. the set of all finite sequences with terms zero or one and first term zero.

Inductively we produce families $\left(\Gamma_{\delta}\right)_{\delta \in \mathscr{D}}$ of uncountable subsets of $\Gamma$ and $\left(W_{\delta}\right)_{\delta \in \mathscr{D}}$ of clopen subsets of $S$ such that

(i) If $\delta_{1}<\delta_{2}, \Gamma_{\delta_{2}} \subset \Gamma_{\delta_{1}}$.

(ii) If $|\delta|>0, \operatorname{diam}\left\{T^{*} \mu_{\gamma}: \gamma \in \Gamma_{\delta}\right\}<\frac{1}{|\delta|}$.

(iii) For $\delta \in \mathscr{D}$ and for all $\gamma_{0} \in \Gamma_{\delta \cup\{0\}}, \gamma_{1} \in \Gamma_{\delta \cup\{1\}}$

$$
\left(\mu_{\gamma_{0}}-\mu_{\gamma_{1}}\right)\left(W_{\delta}\right)>\frac{1}{2} .
$$

We set $\Gamma_{0}=\Gamma$ and we will show how we get $W_{0}$, and $\Gamma_{(0,0)}$, $\Gamma_{(0,1)}$.

We choose an uncountable family

$$
\left(\gamma_{j}^{0}, \Gamma_{j}^{1}\right)_{j \in J}
$$

of pairwise disjoint two-point subsets of $\Gamma$. For each $j$ in $J$ we choose $W_{j}$ clopen subset of $S$ such that

$$
\left(\mu_{\gamma_{j}^{0}}-\mu_{\gamma_{j}^{1}}\right)\left(W_{j}\right)>\frac{1}{2} .
$$

Since the set of the clopen subsets of $S$ is countable there exists an uncountable subset $I$ of $J$ and $W_{0}$ such that $W_{j}=W_{0}$ for all $j \in I$. Also since $K$ is separable there exists an uncountable subset $\Gamma_{(0,0)}$ of $\left(\gamma_{j}^{0}\right)_{j \in I}$ and a subset $\Gamma_{(0,1)}$ of $\left(\gamma_{j}^{1}\right)_{j \in I}$ such that

$$
\operatorname{diam}\left[\left(T^{*}\left(\mu_{\gamma}\right)\right)_{\gamma \in \Gamma_{(0,0)}}\right]<1 \text { and } \operatorname{diam}\left[\left(T^{*}\left(\mu_{\gamma}\right)\right)_{\gamma \in \Gamma_{(0,1)}}\right]<1 .
$$


This completes the inductive construction for the first level. The $n$th level's construction follows from the same arguments and we leave it to the reader.

Next we choose $\gamma_{\delta} \in \Gamma_{\delta}$ for all $\delta \in \mathscr{D}$. Notice that condition (ii) implies that if $d$ is an infinite branch of $\mathscr{D}$ the sequence

$$
\left(T^{*} \mu_{\gamma_{d \mid n}}\right)_{n \in N}
$$

norm converges to some $x_{d}$ element of $K$. Further for all $\alpha \in \mathscr{A}$ if

$$
x_{d \mid n}=T^{*} \mu_{\gamma_{d \mid n}}
$$

$\lambda_{\alpha}^{\left(x_{d \mid n}\right)}$ converges to $\lambda_{\alpha}^{\left(x_{d}\right)}$. Hence the sequence

$$
\left(\mu_{\gamma_{d \mid n}}\right)_{n \in N}
$$

$w^{*}$-converges to some $\mu_{d}$ and $\mu_{d}=\mu_{x_{d}}$; therefore $\mu_{d} \in M_{K}$.

Now let $d_{0}, d_{1}$ be infinite branches of $\mathscr{D}$ with $d_{0} \neq d_{1}$. Set $n_{0}=\max \left\{n: d_{0}\left|n=d_{1}\right| n\right\}$. Without loss of the generality assume that $d_{0}\left(n_{0}+1\right)=0$ and $d_{1}\left(n_{0}+1\right)=1$. Then

$$
\left(\mu_{d_{0}}-\mu_{d_{1}}\right)\left(W_{d \mid n_{0}}\right)=\left[w^{*}-\lim _{n \rightarrow \infty}\left(\mu_{\gamma_{d_{0} \mid n}}-\mu_{\gamma_{d_{1} \mid n}}\right)\right]\left(W_{d \mid n_{0}}\right) \geq \frac{1}{2} .
$$

Therefore $\left\|\mu_{d_{0}}-\mu_{d_{1}}\right\| \geq \frac{1}{2}$.

This proves the claim and the proof of the lemma is complete.

4.10. Definition. (i) A bounded $\delta$-bush $\left(x_{\alpha}\right)_{\alpha \in \mathscr{A}}$ is said to be Dunford-Pettis (D-P) if the corresponding operator $T: L^{1}(S, \lambda) \rightarrow X$ is a Dunford-Pettis operator i.e. it carries weakly compact sets into norm compact sets.

(ii) A $\delta$-approximate bush $\left(x_{\alpha}\right)_{\alpha \in \mathscr{A}}$ is said to be (D-P) if the corresponding averaged bach bush $\left(\tilde{x}_{\alpha}\right)_{\alpha \in \mathscr{A}}$ is a D-P bush.

4.11. REMARK. (i) It is known [D-U] that an operator $T$ is a D-P operator if and only if the corresponding martingale is Cauchy in Pettis norm. That is

$$
\lim _{n, m} \sup _{x^{*} \in B_{X^{*}}} \int\left|x^{*} \varphi_{n}-x^{*} \varphi_{m}\right| d \lambda=0 .
$$

As it is noticed in [A-P] if $\left(x_{\alpha}\right)_{\alpha \in \mathscr{A}}$ is a $\delta$-approximate bush such that for all $\alpha \in \mathscr{A}$

$$
\left\|\sum_{\beta \in S_{\alpha}} \sigma_{\beta} \lambda_{\beta} y_{\beta}\right\|<\varepsilon_{|\alpha|}
$$


for all $\sigma_{\beta}= \pm 1$, then the operator $T$ corresponding to the averaged back bush $\left(\tilde{x}_{\alpha}\right)_{\alpha \in \mathscr{A}}$ is a D-P operator.

(ii) Another class of D-P bushes is that of $\delta$-approximate bushes such that the norm and weak topologies on $\overline{\mathrm{co}}\left(\tilde{x}_{\alpha}\right)_{\alpha \in \mathscr{A}}$ coincide. In such a case it is rather easy to see that the corresponding operator is a D-P operator.

4.12. THEOREM. If $W$ is a convex closed non-R.N.P. subset of a Banach space $X$ then there exists $\left(x_{\alpha}\right)_{\alpha \in \mathscr{A}} \delta$-approximate DunfordPettis bush contained in $W$ and defining a C.F.D.S.D.

Proof. If $W$ contains a non-dentable subset $L$ with the P.C.P. then there exists a $\delta$-approximate bush $\left(x_{\alpha}\right)_{\alpha \in \mathscr{A}}$ in $L$ defining a C.F.D.S.D. and such that the norm and weak topologies on $\overline{\mathrm{co}}\left(\tilde{x}_{\alpha}\right)_{\alpha \in \mathscr{A}}$ coincide (Theorem 3.7). As we mentioned in the previous remark this implies that $\left(x_{\alpha}\right)_{\alpha \in \mathscr{A}}$ is a D-P approximate bush.

If R.N.P. is equivalent to P.C.P. on the subsets of $W$, using Theorem 2.7 and the first part of Remark 4.11 we get the desired bush.

4.13. Corollary. If $W$ is a convex closed non R.N.P. subset of a Banach space $X$ with an unconditional basis and R.N.P. is equivalent to P.C.P. on the subsets of $W$ then $W$ contains a $\delta$-approximate bush $\left(x_{\alpha}\right)_{\alpha \in \mathscr{A}}$ defining a C.F.D.S.D. and which is D-P and almost unconditional.

Proof. The proof is simply a combination of the inductive constructions in the proofs of Theorem 2.7 and 2.13.

We also need the following result from [G-R]:

4.14. Proposition. Let $T: L^{1}(\lambda) \rightarrow X$ be a bounded linear $D$ $P$ operator. Then there exist $\delta>0$ and $a \delta$-dyadic bush $\left(f_{t}\right)_{t \in \mathscr{D}}$ contained in the densities of $\lambda$ such that $T\left[\overline{\mathrm{co}}\left(f_{t}\right)_{t \in \mathscr{D}}\right]$ is a relatively norm-compact set.

We pass now to the statement and the proof of the main result of this section.

4.15. THEOREM. Let $W$ be a closed convex non-R.N.P. subset of a Banach space $X$ such that one of (i), (ii), (iii) is true:

(i) The R.N.P. is not equivalent to the P.C.P. on the subsets of $W$.

(ii) $X$ has an unconditional basis. 
(iii) $W$ is a subset of the positive cone of $L^{1}(\lambda)$.

Then there exists a closed convex subset $K$ of $W$ with a $\mathscr{P a l}$-representation.

Proof. In each one of these three cases the set $W$ contains an adequate $\delta$-approximate bush $\left(x_{\alpha}\right)_{\alpha \in \mathscr{A}}$ (Proposition 4.8). Furthermore we may assume that $\left(x_{\alpha}\right)_{\alpha \in \mathscr{A}}$ is a Dunford-Pettis bush (Theorem 4.12, Corollary 4.13). We set $K=\overline{\mathrm{co}}\left(\tilde{x}_{\alpha}\right)_{\alpha \in \mathscr{A}}$ and we show that $M_{K}$ is non-separable.

Indeed, let $T: L^{1}(S, \lambda) \rightarrow X$ be the corresponding D-P operator. Consider $L^{1}(S, \lambda)$ as a subset of $M(S)$ and set $L=\overline{\mathrm{co}}\left(f_{t}\right)_{t \in \mathscr{D}}$ where $\left(f_{t}\right)_{t \in \mathscr{D}}$ is the dyadic bush of Proposition 4.14. Clearly $L$ is a subset of $M_{K}$ and since $T[L]$ is a norm relatively compact set the $w^{*}$ closure of $L$ in $M(S), \bar{L}^{w^{*}}$, is also contained in $M_{K}$. Since $L$ is a non-dentable set we get that $\bar{L}^{w^{*}}$ is non-separable [R]. The desired result follows now from Lemma 4.9 and the proof is complete.

4.16. REMARK. The fact that in each one of the three cases the set $W$ contains a convex closed subset without extreme points is already known.

Indeed, the first case follows from Rosenthal's proof [R] for Schachermayer's theorem [S2] on the equivalence of R.N.P. and K.M.P. for strongly regular sets. Case (ii) is contained in [S2] and partially in [R-W]. Case (iii) is due to Caselles [C].

4.17. Remark. Certain results of the paper can be stated and proved in a more general setting.

Indeed, everything related to Banach spaces with an unconditional basis can be considered for Banach spaces with an unconditional skipped-blocking decomposition [R-W].

Also instead of the positive cone of $L^{1}(\lambda)$ we may consider Banach lattices $E$ such that $E_{+}$is a solid subset of $E_{+}^{* *}$.

We conclude this section with the following result related to $\mathscr{P} a \ell$ representation in convex $w^{*}$-closed subset of dual Banach spaces.

4.18. Corollary. If $L$ is a convex $w^{*}$-closed non-R.N.P. subset of a dual Banach space $X^{*}$ then it contains a separable convex closed $K$ that has a $\mathscr{P} a$-representation.

Proof. Choose $L^{\prime}$ a $w^{*}$-compact convex non-R.N.P. subset of $L$. Then as Schachermayer proves [S2, Corollary 2.10] either $L^{\prime}$ is $w^{*}$ 
strongly regular or it contains a closed convex subset $K$ affinely homeomorphic to $D(\lambda)$, the set of densities in $L^{1}([0,1], \lambda)$.

In the last case the desired result follows from Theorem 4.15(iii). In the first case the result follows from case (i) of Theorem 4.15 and the main result of $[\mathbf{R}]$. Hence the proof is complete.

4.19. RemarK. Huff-Morris in [H-M] proved that the R.N.P. is equivalent to the K.M.P. for dual Banach spaces. Schachermayer in [S2, Corollary 2.11] proved the analogue of the statement of Corollary 4.18 for the equivalence of the R.N.P. and the K.M.P.

Acknowledgment. We wish to thank Dr. M. Petrakis for some valuable discussions during the preparation of the paper, especially for bringing to our attention Proposition 4.14.

\section{REFERENCES}

[A-P] S. Argyros and M. Petrakis, A property of non-strongly regular operators, Proceedings of the International Conference in Banach spaces at Strobl (to appear).

[B] J. Bourgain, Dentability and finite-dimensional decompositions, Studia Math., 67 (1980), 135-148.

[B-R] J. Bourgain and H. P. Rosenthal, Geometrical implications of certain finite-dimensional decompositions, Bull. Soc. Math. Belg., 32 (1980), 5782.

[Bo] R. D. Bourgin, Geometric Aspects of Convex Sets with the Radon-Nikodým Property, Lecture Notes in Mathematics Vol. 993, Springer-Verlag, New York/Berlin (1983).

[C] V. Caselles, $A$ short proof of the equivalence of K.M.P. and R.N.P. in Banach lattices and preduals of von Neumann algebras, Proc. Amer. Math. Soc., 102 (1988), 973-974.

[D-U] J. Diestel and J. J. Uhl, Vector Measures, Math. Surveys, vol. 15, Amer. Math. Soc., Providence, RI, (1977).

[G-G-M-S] N. Ghoussoub, G. Godefroy, B. Maurey and W. Schachermayer, Some topological and geometrical structures in Banach spaces, Mem. Amer. Math. Soc., 70 (1987), No. 378.

[G-L-M] N. Ghoussoub, J. Lindenstrauss and B. Maurey, Analytic martingales and plurisubharmonic barriers in complex Banach spaces, Contemp. Math., 85 (1989), 111-129.

[G-M-S] N. Ghoussoub, B. Maurey and W. Schachermayer, Pluriharmonically dentable complex Banach spaces, J. Reine Angew. Math., 402 (1986), 76-127.

[G-R] N. Ghoussoub and H. P. Rosenthal, Martingales, $G_{\delta}$-embeddings and quotients of $L^{1}$, Math. Ann., 264 (1983), 321-332.

[H-M] R. E. Huff and P. D. Morris, Dual spaces with the Krein-Milman property have the Radon-Nikodym property, Proc. Amer. Math. Soc., 49 (1975), 104-108. 
[L-L-T] Bor-Liu Lin, Pei-Kei Lin and S. L. Troyanski, $A$ characterization of denting points of a closed bounded convex set, Longhorn Notes, U. T. Functional Analysis Seminar (1985-1986), The University of Texas at Austin.

[R] H. P. Rosenthal, On the structure of non-dentable closed bounded convex sets, Adv. in Math., 70 (1988), 1-58.

[R-W] H. P. Rosenthal and A. Wessel, The Krein-Milman property and a martingale coordinatization of certain non-dentable convex sets, Pacific J. Math., 136 (1989), 159-182.

[S1] W. Schachermayer, On a theorem of J. Bourgain on finite-dimensional decompositions and the Radon-Nikodym property, Lecture Notes in Mathematics Vol. 1267, Springer-Verlag, New York/Berlin (1987), 96-112. $R$. N. P. and K. M. P. are equivalent for strongly regular sets, Trans. Amer. Math. Soc., 303 (1987), 673-687.

Received September 10, 1990 and in revised form July 2, 1991. This research has been supported by a grant of the University of Crete.

UNIVERSITY OF CRETE

HERAKLEION CRETE

GREECE 


\section{PACIFIC JOURNAL OF MATHEMATICS EDITORS}

V. S. VARADARAJAN (Managing Editor)

University of California

Los Angeles, CA 90024-1555

vsv@math.ucla.edu

Herbert Clemens

University of Utah

Salt Lake City, UT 84112

clemens@math.utah.edu

F. Michael Christ

University of California

Los Angeles, CA 90024-1555

christ@math.ucla.edu

THOMAS ENRIGHT

University of California, San Diego

La Jolla, CA 92093

tenright@ucsd.edu
Nicholas ERcolani

University of Arizona

Tucson, AZ 85721

ercolani@math.arizona.edu

R. FINN

Stanford University

Stanford, CA 94305

finn@gauss.stanford.edu

VAUGHAN F. R. JoNES

University of California

Berkeley, CA 94720

vfr@math.berkeley.edu

STEVEN KeRCKHOFF

Stanford University

Stanford, CA 94305

spk@gauss.stanford.edu
C. C. MOORE

University of California

Berkeley, CA 94720

Martin ScharlemanN

University of California

Santa Barbara, CA 93106

mgscharl@henri.ucsb.edu

Harold Stark

University of California, San Diego

La Jolla, CA 92093

\begin{tabular}{|c|c|c|c|c|}
\hline \multicolumn{5}{|c|}{ ASSOCIATE EDITORS } \\
\hline R. ARENS & $\begin{array}{l}\text { E. F. BECKENBACH } \\
(1906-1982)\end{array}$ & B. H. NeumanN & $\begin{array}{c}\text { F. WoLF } \\
(1904-1989)\end{array}$ & K. YoshID \\
\hline \multicolumn{5}{|c|}{ SUPPORTING INSTITUTIONS } \\
\hline \multicolumn{2}{|c|}{ UNIVERSITY OF ARIZONA } & \multicolumn{3}{|c|}{ UNIVERSITY OF OREGON } \\
\hline \multicolumn{2}{|c|}{ UNIVERSITY OF BRITISH COLUMB } & \multicolumn{3}{|c|}{$\begin{array}{l}\text { UNIVERSITY OF SOUTHERN C } \\
\text { STANFORD UNIVERSITY }\end{array}$} \\
\hline \multicolumn{2}{|c|}{ CALIFORNIA INSTITUTE OF TECHNOLOGY } & \multirow{2}{*}{\multicolumn{3}{|c|}{$\begin{array}{l}\text { STANFORD UNIVERSITY } \\
\text { UNIVERSITY OF HAWAII }\end{array}$}} \\
\hline \multicolumn{2}{|c|}{ UNIVERSITY OF CALIFORNIA } & & & \\
\hline \multicolumn{2}{|c|}{ MONTANA STATE UNIVERSITY } & \multicolumn{3}{|c|}{ UNIVERSITY OF TOKYO } \\
\hline \multicolumn{2}{|c|}{ JNIVERSITY OF NEVADA, RENO } & \multicolumn{3}{|c|}{ UNIVERSITY OF UTAH } \\
\hline \multicolumn{2}{|c|}{ NEW MEXICO STATE UNIVERSITY } & \multicolumn{3}{|c|}{ WASHINGTON STATE UNIVERSITY } \\
\hline \multicolumn{2}{|c|}{ OREGON STATE UNIVERSITY } & \multicolumn{3}{|c|}{ UNIVERSITY OF WASHINGTON } \\
\hline
\end{tabular}




\section{PACIFIC JOURNAL OF MATHEMATICS}

Volume $155 \quad$ No. $1 \quad$ September 1992

Characterization of modular correspondences by geometric properties 1 Allan Russell AdLer

Representations of convex nondentable sets

SPIROS ARGYROS and IRENE DELIYANNI

Isomorphisms of spaces of continuous affine functions

CHO-Ho CHU and HENRY BRUCE COHEN

Universal classes of Orlicz function spaces

FRANCISCO LUIS HERNÁNDEZ RODRÍGUEZ and CESAR RUIZ

Asymptotic behavior of the curvature of the Bergman metric of the thin 99 domains

KANG-TAE KIM

Quadratic central polynomials with derivation and involution

CHARLES PHILIP LANSKI

Nonsplit ring spectra and products of $\beta$-elements in the stable homotopy of Moore spaces

\section{JIN KUN LIN}

Orientation and string structures on loop space

DENNIS MCLAUGHLIN

Homomorphisms of Bunce-Deddens algebras

\section{CORNEL PASNICU}

Certain $C^{*}$-algebras with real rank zero and their corona and multiplier 169 algebras. Part I

SHUANG ZHANG

Correction to: "On the density of twistor elementary states"

Michael G. Eastwood and A. M. Pilato 